University of Nebraska - Lincoln

DigitalCommons@University of Nebraska - Lincoln

Faculty Publications from the Harold W. Manter Laboratory of Parasitology

Parasitology, Harold W. Manter Laboratory of

2003

\title{
Fleas and Lice of Mammals in New Mexico
}

\author{
Paulette L. Ford \\ United States Department of Agriculture, Rocky Mountain Research Station, Albuquerque Lab \\ Richard Fagerlund \\ University of New Mexico \\ Donald W. Duszynski \\ University of New Mexico, eimeria@unm.edu \\ Paul Polechla \\ University of New Mexico
}

Follow this and additional works at: https://digitalcommons.unl.edu/parasitologyfacpubs

Part of the Parasitology Commons

Ford, Paulette L.; Fagerlund, Richard; Duszynski, Donald W.; and Polechla, Paul, "Fleas and Lice of Mammals in New Mexico" (2003). Faculty Publications from the Harold W. Manter Laboratory of Parasitology. 198.

https://digitalcommons.unl.edu/parasitologyfacpubs/198

This Article is brought to you for free and open access by the Parasitology, Harold W. Manter Laboratory of at DigitalCommons@University of Nebraska - Lincoln. It has been accepted for inclusion in Faculty Publications from the Harold W. Manter Laboratory of Parasitology by an authorized administrator of DigitalCommons@University of Nebraska - Lincoln. 


\section{USDA}

United States

Department

of Agriculture

Forest Service

Rocky Mountain

Research Station

General Technical

Report RMRS-GTR-123

February 2004

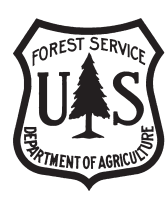

\section{Fleas and Lice} of Mammals in

New Mexico

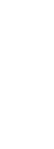

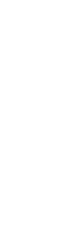

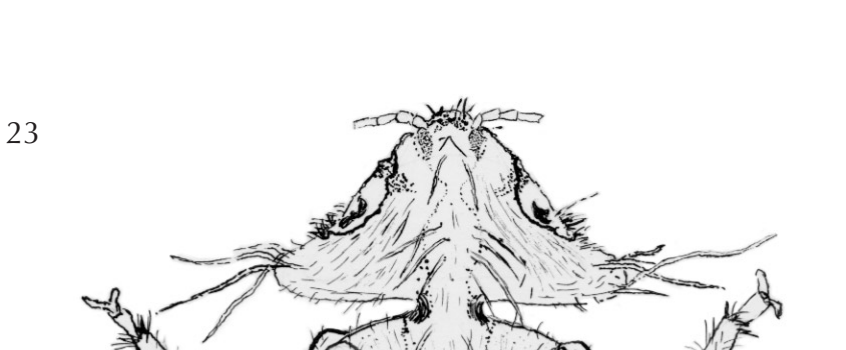

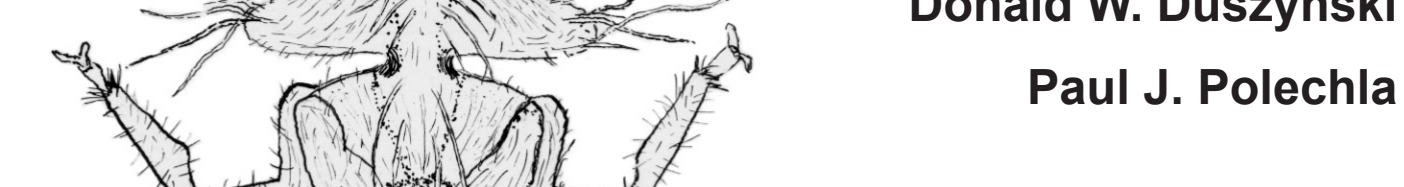

Paulette L. Ford Richard A. Fagerlund Donald W. Duszynski Paul J. Polechla

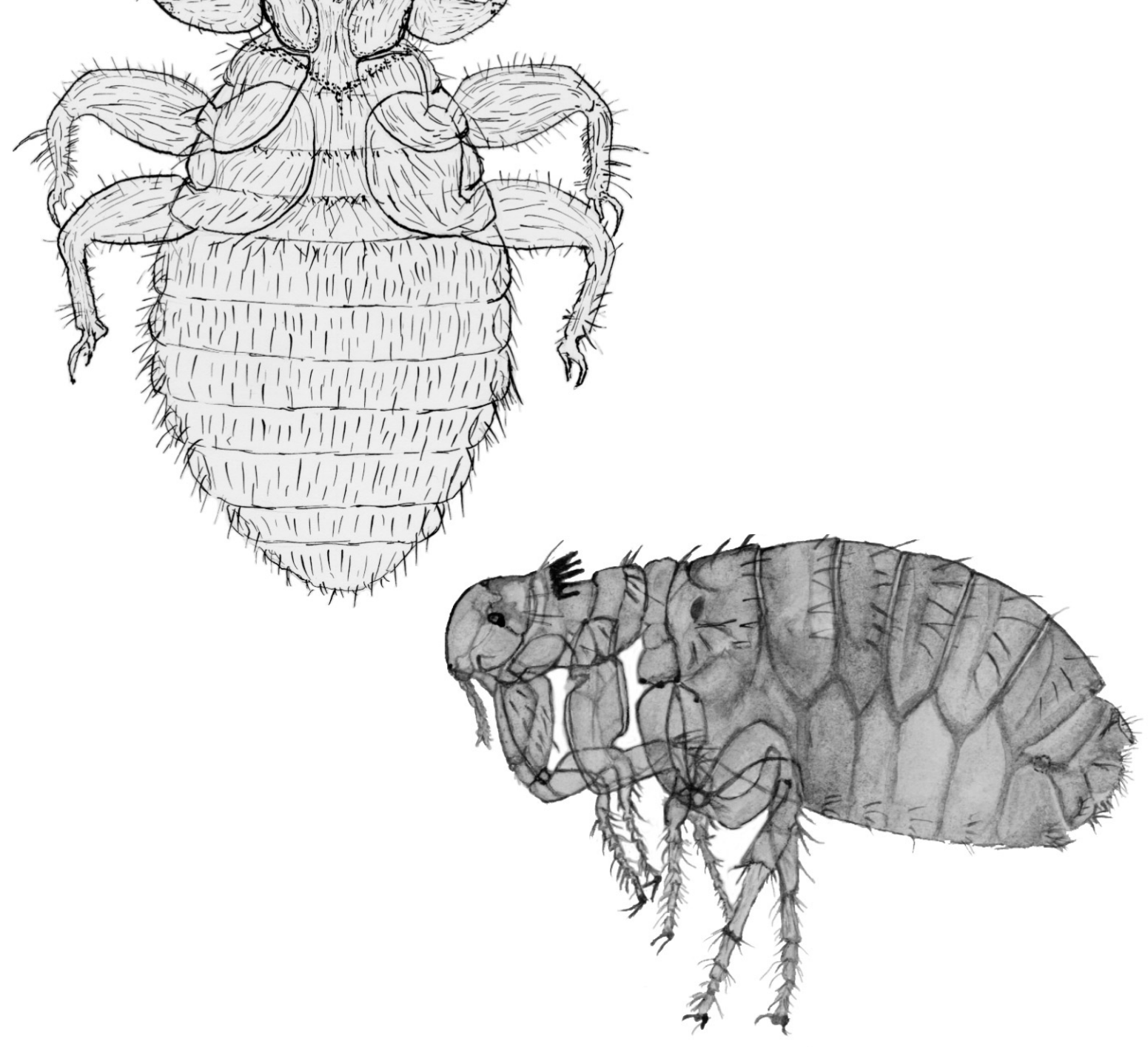


Ford, Paulette L.; Fagerlund, Richard A.; Duszynski, Donald W.; Polechla, Paul J. 2004. Fleas and lice of mammals in New Mexico. Gen. Tech. Rep. RMRS-GTR-123. Fort Collins, CO: U.S. Department of Agriculture, Forest Service, Rocky Mountain Research Station. 57 p.

\section{Abstract}

All available records are compiled for three orders of ectoparasites of mammals in New Mexico: fleas (Siphonaptera), sucking lice (Anoplura), and chewing lice (Mallophaga). We have drawn from records at the University of New Mexico's Museum of Southwestern Biology, the Vector Control Program of the New Mexico Environment Department, the Environmental Health Department of the City of Albuquerque, and several private collections. We list 99 species of fleas, 27 species of sucking lice, and two species of chewing lice. Included are appendices that list recorded ectoparasite species and their hosts in New Mexico and the counties associated with host ectoparasite infestations. We report at least four new state host records for fleas.

Keywords: Anoplura, chewing lice, ectoparasite, flea, lice, louse, Mallophaga, New Mexico mammalian hosts, Siphonaptera, sucking lice

\section{The Authors}

Paulette Ford is a Research Ecologist with the USDA Forest Service, Rocky Mountain Research Station, in Albuquerque, NM. She holds an M.S. in biology from the University of New Mexico and a Ph.D. in Renewable Natural Resources from the University of Arizona. She has worked extensively in Latin America and the American Southwest on research ranging from the systematics of parasites and amphibians to small mammal and amphibian community structures in deserts, grasslands, and tropical deciduous forests. Richard Fagerlund is an Integrated Pest Management Coordinator with the University of New Mexico. He currently has a newspaper column in the Albuquerque Tribune on pest control and is the author of four books on insects. Donald Duszynski is a Professor of Biology at the University of New Mexico. He has an M.S. and Ph.D. from the Department of Zoology at Colorado State University. He has been at UNM since 1970 and specializes in endoparasites of wild mammals, especially the coccidian or parasitic protists. Paul Polechla is a Research Associate Professor at the University of New Mexico in the biology department's Museum of Southwestern Biology Mammal Division. He earned his M.S. at Eastern New Mexico University and his Ph.D. at the University of Arkansas. He was a member of the Southwestern Zoonotic Disease Program (1996-2000) studying hantavirus and deer mice (Peromyscus maniculatus) ecology. His interests are mammalian ecology with an emphasis on predator-prey and host-parasite relationships.

cover illustrations by: Johnna Autumn Strange. Louse (upper), flea (lower).

You may order additional copies of this publication by sending your mailing information in label form through one of the following media. Please specify the publication title and series number.

Fort Collins Service Center

$\begin{aligned} \text { Telephone } & (970) \text { 498-1392 } \\ \text { FAX } & (970) 498-1396 \\ \text { E-mail } & \text { rschneider@fs.fed.us } \\ \text { Web site } & \text { http://www.fs.fed.us/rm } \\ \text { Mailing address } & \text { Publications Distribution } \\ & \text { Rocky Mountain Research Station } \\ & \text { 240 West Prospect Road } \\ & \text { Fort Collins, CO 80526 }\end{aligned}$

Rocky Mountain Research Station

Natural Resources Research Center

2150 Centre Avenue, Building A

Fort Collins, CO 80526 


\section{Contents}

Acknowledgments . . . . . . . . . . . . . . . . . . . . .

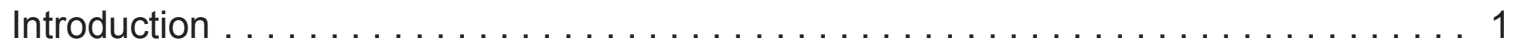

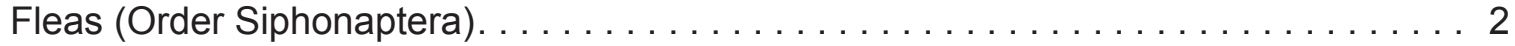

General ............................... 2

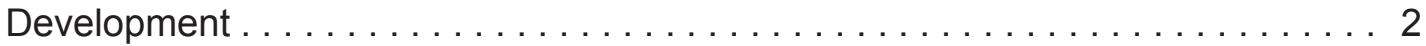

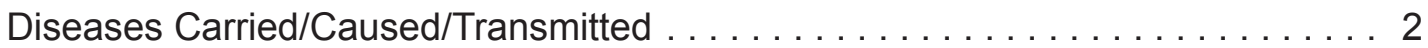

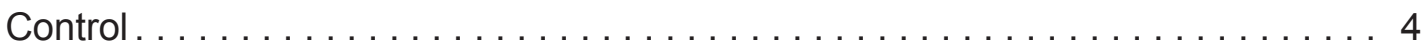

Sucking Lice (Order Anoplura) $\ldots \ldots \ldots \ldots \ldots \ldots \ldots \ldots \ldots \ldots \ldots \ldots \ldots \ldots \ldots \ldots \ldots$

General . . . . . . . . . . . . . . . . . . . . . . . . . . . . . . 4

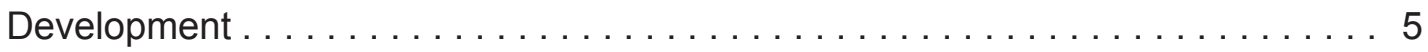

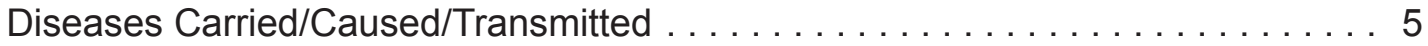

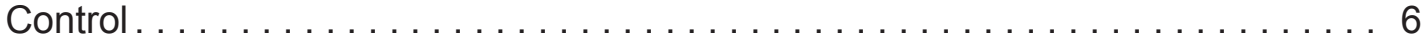

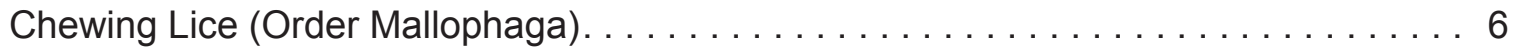

General . . . . . . . . . . . . . . . . . . 6

Development . . . . . . . . . . . . . . . . . $6 \ldots \ldots \ldots$

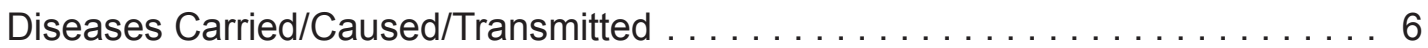

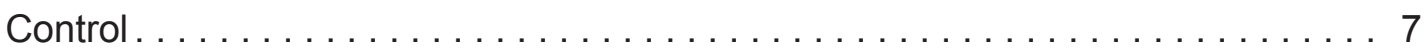

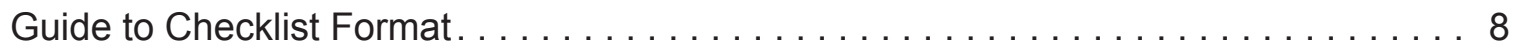

Host List—New Mexico Mammals and Their Ectoparasites . . . . . . . . . . . . . 9

Order Artiodactyla . . . . . . . . . . . . . . . . . . . . . . . 9

Order Carnivora . . . . . . . . . . . . . . . . . . . . . . 9

Order Chiroptera . . . . . . . . . . . . . . . . . . . . . . . 12

Order Insectivora . . . . . . . . . . . . . . . . . . . . 13

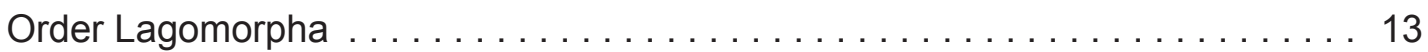

Order Perissodactyla . . . . . . . . . . . . . . . . . 16

Order Primates . . . . . . . . . . . . . . . . . . . . . . 16

Order Rodentia . . . . . . . . . . . . . . . . . . . . . . . . . 17

References . . . . . . . . . . . . . . . . . . . . . . . . 39

Appendix A. New Mexico Ectoparasites and Their Mammal Hosts. . . . . . . . . . . 42

Order Siphonaptera (Fleas). . . . . . . . . . . . . . . . . . . . . . . 42

Order Anoplura (Sucking Lice) . . . . . . . . . . . . . . . . . . . 49

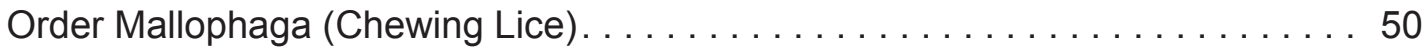

Appendix B. New Mexico County Records . . . . . . . . . . . . . . . . . . . . . 51

Fleas (Siphonaptera) . . . . . . . . . . . . . . . . . . . . . 51

Lice (Order Anoplura and Order Mallophaga) . . . . . . . . . . . . . 56 


\section{Acknowledgments}

We wish to thank the following people and institutions: Sandra Brantley for her extensive work with the manuscript and the arthropod collections; Lee Couch for compiling the data, editing, and organizing the final manuscript; Dawn Chen Sun for reviewing the reference material used in the manuscript; Clifford Crawford, Lane Eskew, and Deborah Finch for reviewing multiple drafts of the manuscript and making many valuable suggestions; and Terrence Enk, New Mexico Department of Game and Fish, for specific consultation. Thanks to Ted Brown and Pamela Reynolds, New Mexico Environment Department; New Mexico Environment Department's Vector Control Program; Rudy Bueno, City of Albuquerque Environmental Health Department; Cheryl Parmenter and Kim Heckscher-Decker, Department of Biology, UNM and the Sevilleta Long Term Ecological Research (LTER) Program; Dwayne Salazar, DJ's Pest Control, Albuquerque, NM; and David C. Lightfoot, Sevilleta LTER, Jornada LTER, and Bandelier National Monument for supplying information, specimens, and identifications. We also thank Terry Yates, principal investigator of the small mammal hantavirus studies and Jon Dunnum, Brian Frank, W. Scott Knapp, Kimberly Leuthner, and Todd Meinecke for assistance in trapping small mammals that supplied specimens; Peggy Case for contributing ectoparasites from lagomorphs; and the Bureau of Land Management, the National Institutes of Health, Centers for Disease Control and Prevention (CDC), and the Bureau of Indian Affairs for funding mammal studies that contributed data to this document. This manuscript is dedicated to Michael J. Patrick, assistant professor of biology at Pennsylvania State University, who contributed many of the host animals and flea descriptions that are included in the checklist. Dr. Patrick died 10 March 2000. 


\title{
Fleas and Lice From Mammals in New Mexico
}

\author{
Paulette L. Ford, Richard A. Fagerlund, \\ Donald W. Duszynski, and Paul J. Polechla
}

\section{Introduction}

The purpose of this work is to provide baseline data of what is known and can be documented about the fleas and lice of New Mexico mammals. Within that context, we summarize the publications on this topic, document the fleas and lice that are accessioned into the Arthropod Division of the University of New Mexico's Museum of Southwestern Biology (UNM-MSB), and provide some general information about the biology of fleas and lice and the potential disease agents they may transmit to other mammals in New Mexico, including humans. Since infestations of fleas, sucking lice, and chewing lice in humans, domesticated animals, and wildlife may lead to discomfort, debilitating disease, and/or death, this information has implications for, but not limited to, federal, state, and private land managers, scientists, public health officials, and the general public.

New Mexico has the distinction of having one of the highest diversities of land mammals in the United States. There are approximately 150 extant, native mammal species representing eight orders, 25 families, and 71 genera in the state (Frey and Yates 1996). The potential for flea and louse diversity is, therefore, very great. In addition, when non-native mammals are introduced into the state or increase their range(s) from adjacent states, they bring with them their ectoparasites that may be transmitted to endemic species.

In this manuscript we compile all available records for three orders of ectoparasites of mammals in New Mexico: fleas (Siphonaptera), sucking lice (Anoplura), and chewing lice (Mallophaga). There is a lack of information about these ectoparasites, due to the low numbers of the host species studied and to the fact that not all areas of the state have been sampled thoroughly for fleas. Although there is some information on fleas from certain parts of the state, such as Santa Fe County, much less is known about the lice in New Mexico. There are few records of chewing lice from this state's mammals, and most of the recent records of sucking lice have come from small mammal population studies at the Sevilleta National Wildlife Refuge (SNWR) near Socorro, New Mexico.
Of the 150 mammal species in New Mexico that potentially act as host to one or more species of fleas and lice, only 82 are documented to host these ectoparasites. These reports are widespread temporally and geographically, and most represent only one collection event from one locality. A total of 99 species of fleas, 27 species of sucking lice, and two species of chewing lice are known and documented from New Mexico mammals. However, should every mammal species be thoroughly surveyed throughout its known range, the number of flea and lice species on New Mexico mammals would clearly be much higher than this. For example, we already have documented that at least 29 New Mexico mammals have 10 or more species of fleas recorded from them. For these reasons, it is clear that the study of the mammalian fleas and lice parasitizing New Mexico mammals is in need of attention.

Vertebrate biologists, who work in the field in New Mexico, can play a pivotal role in our understanding of the diversity and distribution of ectoparasites infesting this state's mammal (and other terrestrial vertebrate) populations by properly collecting specimens from study animals. The methods for collecting and preserving ectoparasites in the field are simple yet important:

1. Collect the organism(s) from the surface of the host and place them in a vial or other container with 70 percent ethanol or similar alcohol (see Gardner 1996 and Whitaker 1982 for more specific details).

2. Using a No. 2 pencil or indelible (India) ink pen, write the following information on a small label to be placed into the collection vessel: careful and correct identity of the host animal, its precise location of collection, the sex of the animal and its approximate age (juvenile, adult), the date ( $\mathrm{mm} / \mathrm{dd} / \mathrm{yy})$, and your name and collection number (if any).

3. Send or take specimens to the Arthropod Division, UNM-MSB. If possible, the host animal (symbiotype host, see Frey and others 1992; Brooks 1993) should be collected and also taken to the Mammal Division, UNM-MSB, or placed in another accredited museum.

For each of the three orders of ectoparasites covered in this annotated list, we present information on 
their general biology, their development, diseases they can carry and/or transmit, and the means of controlling them. Mammalian hosts from which we have reliable ectoparasite records are grouped alphabetically by order, and within their order, by family, genus, and species. Under each host species is a list of the specific type of ectoparasites known, the New Mexico counties in which they were collected, the UNM-MSB voucher number if available, any remarks that are appropriate to that parasite or its host, and pertinent reference material(s).

\section{Fleas (Order Siphonaptera)}

\section{General}

Fleas are small, wingless, hematophagous (blood feeding) insects that are compressed laterally and range in size from about 1 up to several millimeters in length. The head is roughly triangular in shape and usually bears a pair of conspicuous black eyes. The entire body, including legs, is covered with bristles and small spines. Approximately 2,500 species and subspecies belonging to 239 genera have been named to date (Roberts and Janovy 2000; Service 2000). They are all ectoparasites of warm-blooded (homiothermic) vertebrate hosts (birds, mammals), and both male and female adults feed on blood. About 94 percent of known species infest mammals, with the remainder infesting birds. Some fleas are highly specific, known from only one host species (Thomas 1996). However, most flea species have one or two preferred host species, others are more euryxenous (generalists) in their feeding preference and are known to parasitize hosts of a particular genus or family of hosts, and still others are cosmopolitan, feeding on virtually any vertebrate host they should chance upon (Thomas 1996). Conversely, some host species are known to harbor only one flea species while, at the other extreme, a few host species have been found to host more than 50 flea species throughout their range (Thomas 1988).

Fleas, like all insects, have three pairs of legs, but theirs are powerful and specialized for jumping, especially the longer hind legs. Some fleas can jump more than 100 times their body length and/or can execute a standing jump more than 120 times their height. In doing this, they can reach an acceleration of 140 times gravity in little more than a millisecond. How they can accomplish such extraordinary feats is not completely understood (Roberts and Janovy 2000). We do not understand yet the exact necessity of possessing such ability (e.g., avoiding predation, moving from host to host, demonstrating good genes in sexual selection).

\section{Development}

Fleas are all holometabolous, i.e., they undergo a complete metamorphosis with (usually) four distinct developmental stages between the egg and the adult. Egg laying behavior of adult fleas is almost always adapted and co-evolved to the behavior of the host upon which it lives (Kim 1985). Usually, females ready to oviposit leave the host to deposit eggs in the host's immediate or general territory (nest or burrow); in a few species, the female remains on the host, but her smooth eggs usually fall to the ground. Off the host, eggs are laid in/ near cracks and crevices among dust, dirt, and debris. Generally, eggs hatch in a relatively short period of time (2-21 days), but this depends on environmental conditions (especially rodent burrow temperature and humidity) (Thomas 1996; Roberts and Janovy 2000; Service 2000). The stage that hatches from the egg is called larva ( $1^{\text {st }}$ instar) because it does not look like the adult; it later will molt twice producing successive instars. Each of the three instars usually requires some blood in its diet, but this is not taken directly from feeding on a host. Rather, they ingest dried feces from adult fleas along with other debris from their immediate environment (fur, feathers) or, in some extreme cases, feed on fecal blood as it passes directly from the anus of their mother or another feeding adult, while they are attached to that adult's abdomen (Thomas 1996). The $3^{\text {rd }}$ instar flea larva will spin a silken cocoon, or pupal case, often embedded with surrounding detritus, and enter the pupal stage. The pupa remains within its case and undergoes metamorphosis to the adult body form; it stays within the cocoon in the adult form--sometimes for weeks, months, or even up to one year--until certain host recognition factors stimulate it to leave the cocoon.

Upon hatching, the adult fleas must take a blood meal before mating and egg production occurs. In some species, certain growth hormones in the blood of the host may trigger the fleas to mate and lay eggs. Under adverse conditions (host absence), adult fleas can survive long periods without food (e.g., in nests, tunnels), especially under conditions of high humidity (Roberts and Janovy 2000). Unlike adults, however, larvae cannot tolerate extremes in relative humidity and will die if the humidity is either too high or too low (Service 2000).

\section{Diseases Carried/Caused/Transmitted}

Fleas are primarily a nuisance due to the considerable discomfort, irritation, and annoyance caused by their bites. The most common nuisance flea is the cat flea, Ctenocephalides felis. Other fleas of lesser importance 
are the dog flea, C. canis, and the so-called human flea, Pulex irritans. Fleas of other domesticated animals may be of local importance. Fleas frequently bite humans on the ankles and legs, but at night during sleep, people may be bitten all over the body. Intense itching may result in a person becoming sensitized, and children usually experience greater discomfort than older persons (Service 2000). Some fleas of wild animals are important vectors of disease; these are summarized below.

\section{Plague}

Plague is primarily a disease of wild animals (a zoonosis), especially rodents. Over 200 mammal species have been shown to harbor plague bacteria, with some species being particularly susceptible. Prairie dogs, especially Cynomys gunnisoni, are uniformly susceptible to fatal infections with plague, and large proportions (99 percent) or even entire populations have been destroyed in a single epizootic event (Lechleitner and others 1962; Hubbard and Schmitt 1984).

Plague is caused by a bacterium, Yersinia pestis (syn. Pasturella pestis); it is of Old World origin and throughout history has been referred to as the "Black Death." The profound impact of plague on humans and on human history, more than any other single infectious agent ever, is summarized by Hubbard and Schmitt (1984), Roberts and Janovy (2000), and Marquardt and others (2000).

Plague was first discovered in North America from California ground squirrels (Spermophilus beecheyi) in 1905 (Barnes 1982). It was first detected in native New Mexico rodents in 1938 (Laney 1950), and the first documented human case of plague in New Mexico occurred in 1949 (Rollag and others 1981). In the United States, at least 19 different flea species have been found to bite humans, but species in more than 50 genera are important globally as potential vectors of plague. As of 1982, 18 rodent species (two lagomorphs and nine carnivores) were documented to have been infected by plague in New Mexico (Brown, undated). At least 33 species of flea have tested positive as vectors of sylvatic plague in New Mexico (Fagerlund and others 2001).

The normal cycle of plague transmission is between wild rodents and their fleas in nature and is termed sylvatic, campestral, rural, or endemic plague. When plague bacteria are transmitted to rats living in close association with people, such as in rat-infested slums, fleas (particularly, Xenopsylla cheopis) that normally feed on rats may turn their attention to humans. Rats infected with plague bacteria may develop acute and fatal septicemia. Upon the death of the host, infected fleas will leave this host and feed on humans.
When fleas ingest bacteria along with blood from infected rodents, the bacteria multiply rapidly in the gut of the flea to the extent that their mass blocks the passage of later blood meals through the flea's proventriculus. Thus, when the flea feeds again, the blood it takes in cannot pass the obstruction, becomes contaminated with bacteria, and is regurgitated back into the bite wound. Interestingly, the ability of various flea species to allow rapid growth of $Y$. pestis organisms that block the gut is a deciding factor of the efficacy of the flea as a good vector (Roberts and Janovy 2000).

Plague manifests itself in humans in one of three forms: bubonic, pneumonic, or septicemic. The most common, bubonic, causes swollen lymph nodes in the groin or armpits. These swellings or "buboes" can get as big as chicken eggs and occur in about 75 percent of all human cases during epidemics. Pneumonic plague occurs when the lungs are heavily involved and produces a pneumonia-like condition that is highly contagious to other humans. Septicemic plague is a generalized blood infection, often with little or no prior lymph node involvement (Roberts and Janovy 2000). Humans become infected with plague by being bitten by a Yersinia pestis infected flea or by handling a dead plague infected animal without gloves (NM Dept. of Health, Office of Epidemiology, pers. comm.).

Plague is most common in temperate regions during summer and autumn months and in the tropics during the cooler months. Heat and dryness negatively impact the spread of plague. Campestral plague, that seen in animals of open (rather than wooded) areas, is widespread and common in wild rodents and rabbits of the United States west of the $100^{\text {th }}$ meridian. Human cases in these areas occur only sporadically, often after a person has had contact with wild rodents or rabbits and their fleas. New Mexico has had the highest case rate of human plague in the last decade (Roberts and Janovy 2000). For example, during 1988-2002, a total of 112 human cases of plague were reported from 11 western states. The majority, 97 of the 112 cases (87 percent), were exposed in the four states of Arizona, California, Colorado, and New Mexico, with 48 of the 97 (49 percent) occurring in New Mexico (CDC 2003).

\section{Murine, Flea-Borne or Endemic Typhus}

This form of typhus is caused by Rickettsia typhi $(=R$. mooseri), which is virtually identical to $R$. prowazekii, the typhus-causing organism transmitted to humans by body lice (see below). When ingested by the flea, the rickettsiae multiply in its gut, but unlike plague bacilli, they do not cause blockage. Rather, infection of the vertebrate host occurs when infected feces from the flea is 
rubbed into abrasions or comes into contact with mucous membranes. The rickettsial organisms also can be released when fleas are crushed as they are biting, and then, inadvertently, their contaminated body juices are rubbed into wounds. Murine typhus is essentially a disease of murine rodents, especially Rattus species; it is common in warm climates and also infects a wide range of other small mammals. Murine typhus is transmitted by various fleas including Xenopsylla, Nosopsyllus, and Leptopsyllus species, as well as by the rat louse Polyplax spinulosa and the tropical rat mite Ornithonyssus baco$t i$. Transovarial transmission, when the rickettsial organism passes from an infected female flea to its ovaries, also occurs to transmit the infection from egg to larva to adult (Service 2000). In humans, the rickettsial organism causes a rather mild, febrile illness that lasts about two weeks, accompanied by headache, chills, body pain, and rash. The disease affects elderly people more severely than it does the young. Interestingly, the opossum (Didelphis virginiana), a New Mexico resident (Bermudez and others 1995), also is a reservoir host for murine typhus, and this species is proliferating in many urban and suburban areas, creating the possibility for resurgence of this disease (Roberts and Janovy 2000; Service 2000).

\section{Myxomatosis}

This is a disease of rabbits (Order: Lagomorpha) caused by a Myxoma virus that was native to South America, but it has spread to the USA and the UK. It is transmitted by a number of blood sucking arthropods including fleas, mites, and mosquitoes. The disease can and has caused considerable losses in the domestic rabbit (Oryctolagus cuniculus) industry.

\section{Other Parasites}

The flea Nosopsyllus fasciatus transmits the nonpathogenic kinetoplastid protist Trypanosoma lewisi from rat to rat. Fleas from dogs (C. canis), cats $(C$. felis), and humans ( $P$. irritans) can serve as intermediate hosts of Dipylidium caninum, a tapeworm that is common in cats (Felis catus) and dogs (Canis familiaris) and can be transmitted to humans, especially children. Certain tapeworms of mice and rats also can be transmitted from host to host: Nosopsyllus fasciatus and $X$. cheopis for the rat tapeworm; and Hymenolepis diminuta, X. cheopis, C. felis, C. canis, and P. irritans for the mouse tapeworm H. nana. (Hymenolepis nana is a reasonably common parasite of children who have close contact with flea-infested cats and dogs.) These fleas consume the tapeworm eggs passed in the feces of their vertebrate host; they can act as intermediate hosts by retaining the tapeworm larval stage, the cysticercoid, in their hemocoel until their metamorphosis to the adult form. Humans can then become infected by the inadvertent ingestion of infected fleas. A filarial nematode (Dipetalonema reconditum) that lives in the subcutaneous, connective, and perirenal tissues of dogs can be transmitted from host to host by C. canis and C. felis. The juvenile stage of the worm, called microfilariae, is ingested by fleas during their blood meals, develops to infective stages in the flea's fat body, migrates to the mouthparts, and then passes to the wound when the flea feeds the next time (Roberts and Janovy 2000). Fleas also may transmit Francisella tularensis (tularemia), Rickettsia conori (tick-borne typhus), Coxiella burneti (Q fever), Bartonella henselae (cat-scratch fever), and a few other minor pathogens to humans (Thomas 1996; Service 2000).

\section{Control}

For public health reasons, it is important to control fleas of rodents around our homes and on our dog and cat pets. Places that may harbor fleas within our homes, such as under carpets, floor crevices, and pet bedding materials, should be cleaned often. Various insecticidal flea powders and flea collars with slow-release vapors are effective for ridding dogs and cats of these parasites. Recently, novel, non-chemical devices such as light traps with yellow-green filters to which fleas are attracted have been shown to attract fleas from as far away as 8 m (Dryden and Broce 1993; Roberts and Janovy 2000). Finally, it is important to keep areas where livestock are maintained as free as possible from the buildup of manure, debris, and other litter.

\section{Sucking Lice (Order Anoplura)}

\section{General}

There are only about 500 described species of sucking lice and they are found only on mammals. They have small, wingless bodies that are flattened dorsoventrally. Their head, which is narrower than their prothorax, bears a pair of inconspicuous eyes. Their mouthparts consist of a flexible, sucking tube-like structure called the haustellum, which is armed on the inner surface with minute teeth; the whole structure is retracted into the head when not feeding. Because they introduce their highly modified mouthparts directly into a blood vessel when feeding, they are called true solenophages (Greek for pipe + eating). Several species are of considerable importance 
on domestic animals, and two or three species parasitize humans and can carry disease-producing microbes.

The two species of Anoplura found on humans are Pediculus humanus and Phthirus pubis. Some authorities say there are two distinct forms (subspecies) of $P$. humanus, body lice ( $P . h$. humanus) and head lice $(P$. $h$. capitis), while others contend they are separate species, $P$. capitis and $P$. humanus (=P. corporis) because of subtle structural differences (Busvine 1978; Roberts and Janovy 2000; Service 2000). In either case, it is widely accepted that body lice descended from ancestral head lice after humans began to wear clothes. People who live in tropical climates often have head lice, but because they wear few clothes, body lice are usually absent. Consequently, body lice are much more prevalent in cooler, temperate regions. Although head lice stay closely associated with head hair, especially on the back of the neck and behind the ears, human body lice are unusual among Anoplura in that they spend most of their time in their host's clothing, intimately visiting their host only to take a blood meal. Both forms are highly contagious, especially under conditions of crowding and poor sanitation where people rarely wash or change their clothes. Thus, they are common in jails, refugee camps, trenches during wartime, and after disasters (wars, floods, earthquakes, etc.) where people are forced to live in unsanitary, overcrowded conditions. In the United States, elementary school children are susceptible to head lice when sharing hats, combs, and brushes.

Phthirus pubis, the "crab" louse of humans, is socalled because its middle and hind pair of legs are larger and stouter than the front pair and have massive claws that superficially resemble crabs' pincers. This species is found primarily in the pubic area, although specimens can be found amongst axillary and facial (i.e., beard, mustache, eyebrows, eyelashes) hair. It is transmitted primarily through sexual contact (Service 2000). Infestations also can arise from discarded clothing and infested bedding. Treatment involves the use of 1 percent permethrin solution available from drugstores.

Anoplura tend to be relatively host-specific, but many exceptions occur. For example, P. humanus also can live and breed on pigs (Sus scrofa), while Haematopinus suis of pigs readily feeds on humans. Other Haematopinus species infest cattle (Bos taurus), water buffalo (Bubalus bubalis), horses (Equus caballus), mules (E. caballus $\mathrm{x}$ E. asinus), and donkeys (E. asinus). Different Linognathus species parasitize cattle, sheep (Ovis aries), goats (Capra hircus), and dogs. The latter species may specialize on different regions of their host's body (e.g., legs, head), just as Pediculus species do on humans. Another species, Polyplax spinulosa, infests Rattus species and transmits Rickettsia typhi, the causative agent of murine typhus, also carried by fleas (see above).

\section{Development}

The eggs of sucking lice, called nits, are cemented to the hair of their host, or in the case of body lice of humans, to fibers in the clothes. A female can produce up to 10 eggs per day and may produce 50-150 (head lice), 150-200 (pubic lice), or 200-300 (body lice) eggs in her life. Each egg has an operculum, or cap, at one end, usually with holes to allow the passage of air and to facilitate hatching. Lice have a hemimetabolous life cycle, meaning that the stage hatching from the egg is called a nymph, because it resembles a small adult. Depending on ambient temperature, eggs hatch in about a week, and the three nymphal stages (instars) require one to four weeks to complete their development to the adult. Nymphal stages also take blood meals, like the adults. Body lice usually do not leave their hosts voluntarily, but they are very sensitive to changes in host body temperature. Thus, they will leave their host's body when it cools after death or when the body heats due to high fever.

\section{Diseases Carried/Caused/Transmitted}

Infestation with lice is not life threatening, unless they carry disease-causing microbes. In general, the bites cause red, itching papules which may continue to exude lymph after the bite. Thus, continued scratching may lead to dermatitis and secondary bacterial infections. Years of infestation can lead to darkened, thickened areas of the skin. Allergies that produce severe itching may be caused by repeated inoculation of louse saliva when they bite. If inhaled, louse feces that are dried and become airborne may produce symptoms resembling hay fever. Human body lice can transmit three important diseases.

\section{Louse-Borne, Endemic, or Epidemic Typhus}

Typhus is caused by an obligate, intracellular bacterium Rickettsia prowazekii. Endemic typhus has had a significant impact on human history (see Zinsser 1934). Typhus epidemics usually coincide with anthropogenic events (war, crowding, stress, poverty, mass migrations) that favor heavy and widespread infestations of body lice; mortality during epidemics may approach 100 percent (Roberts and Janovy 2000). The disease manifests a large suite of symptoms including high fever, back- and headache, malaise, vertigo, flushed face, and petechial rashes on the armpits, flanks, chest, 
abdomen, back, and extremities. After about two weeks, the fever drops, profuse sweating begins, and patients become more aware of their condition. At this point, either convalescence results or increased involvement of the central nervous system begins, resulting in death. Interestingly, $R$. prowazekii is a pathogenic, often fatal parasite of the lice themselves. The rickettsial organism invades the louse's gut cells and reproduces to the point that in about 10 days the cells are destroyed, killing the louse. Before death, however, the louse's feces contain massive numbers of rickettsiae, and scratching the louse bite inoculates the organism from the feces into the bite wound. Also, the louse's strong preference for a normal body temperature stimulates it to leave the febrile patient to search for new hosts and, thus, further spreads the disease during epidemics. The rickettsial organisms also can remain infective in louse feces for up to 60 days at room temperature, and humans can become infected with typhus by inhaling dried louse feces. Humans who have survived an initial infection are important reservoir hosts because, although asymptomatic, they are still capable of infecting new lice for years. Both humans and other animal reservoirs could provide the source for new epidemics, here or elsewhere, in the event of invasions, war, famine, or other disasters (Roberts and Jaonvy 2000; Service 2000).

\section{Trench Fever}

This is a relatively uncommon and debilitating but non-fatal disease caused by another rickettsial organism, Bartonella (=Rochalimaea) quintana. It also is transmitted by the human body louse. The disease was first documented during World War I (1914-1918) among soldiers in trenches (thus, the name), and it reappeared again in eastern Europe during World War II (1939-1945). Scattered recent foci of infections have been documented in Bolivia and Mexico, and some cases also have been reported in the United States and Europe, mostly in homeless people (Service 2000).

\section{Relapsing Fever}

This disease of humans, also transmitted by the human body louse, is caused by a spirochete, Borrelia recurrentis. Spirochetes are ingested during a blood meal but only a few pass from the gut of a louse into its body cavity (haemocoel), where they reproduce and reach enormous numbers. The only way humans are infected is when the louse is crushed on the skin, releasing spirochetes to enter the body through abrasions or mucus membranes (Service 2000). Louse-borne relapsing fever apparently has disappeared from the United States (Roberts and Janovy 2000).

\section{Control}

A large variety of over-the-counter products containing insecticides effective against lice are available at most drug and grocery stores. Several, containing permethrin, are incorporated into hair care products like shampoos and cream rinses. Fine-toothed nit combs, hot water washing, machine drying, and/or dry-cleaning clothes will control human body lice. Lice on pets and domestic food animals can be controlled by insecticidal dusts and dips.

\section{Chewing Lice (Order Mallophaga)}

\section{General}

Chewing lice are wingless, dorsoventrally flattened insects with reduced (or no) eyes. There are about 3,000 named species that infest many bird and mammal species. In fact, the majority of known species are parasites of birds. None, however, have any medical significance for humans or their domesticated animals other than being significant pests. They feed primarily on hair or feathers, but some eat sebaceous secretions, mucus, and sloughed epidermal cells, while others eat the eggs and nymphs of their own species, as well as ectoparasitic mites. They also will eat blood when an irritated host scratches itself to the point of causing bleeding. Most chewing lice are only a few millimeters long and have a head that is broader than their prothorax and lack ocelli. Although many fewer species infest mammals than birds, guinea pigs (Cavia spp.), dogs, cats, cattle, horses, mules, asses, sheep, goats, and even Indian elephants (Elaphas maximus) all commonly have chewing lice on them. Irritation of the skin can become severe, especially in the young host.

\section{Development}

The development of chewing lice, from egg to adult, is similar to that seen in the Anoplura.

\section{Diseases Carried/Caused/Transmitted}

Some chewing lice can act as intermediate hosts for a number of endoparasites of mammals and birds. Trichodectes canis, an irritating louse of dogs that can present a severe problem to puppies, is an intermediate host for the double-pored dog tapeworm, Dipylidium caninum, which also can develop in people who may accidentally ingest these insects while playing with or 
petting their pets. The worms are transmitted when the mallophagan picks up the microfilariae by chewing on skin and eating blood from minor wounds.

\section{Control}

Mammals help control biting and other lice by grooming themselves or others. Rodents such as kangaroo rats take dust baths, which may act as a way to rid themselves of chewing lice. Other control measures are similar to those listed above for sucking lice. 


\section{Guide to Checklist Format}

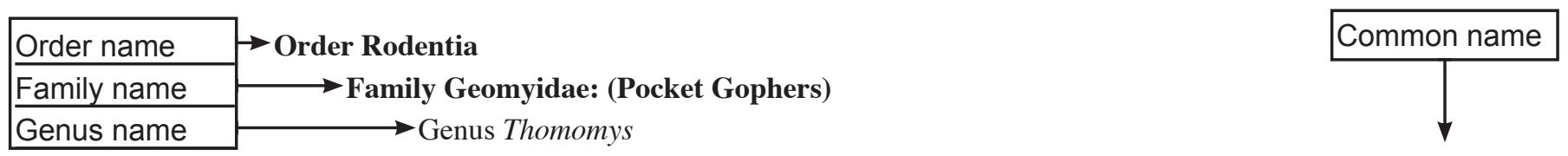

Host: Thomomys bottae (Eydoux and Gervais, 1836). . . . . . . Botta's pocket gopher.

\begin{tabular}{l|l} 
Species name & Siphonaptera: \\
\cline { 2 - 3 } & $\longrightarrow$ ) Foxella ignotus Baker, 1895
\end{tabular}

2) Spicata bottaceps Hubbard, 1943

NM Counties: Sandoval (1); Santa Fe $(1,2)$.

Material Deposited: None.

Reference: Fagerlund and others 2001.

Family Heteromyidae: (Kangaroo Rats, Pocket Mice)

Genus Chaetodipus

Parentheses indicate that the species was named originially under another genus but that subsequent taxonomic revision has placed it in the listed genus.
Host: Chaetodipus hispidus (Baird, 1858) Anoplura: Fahrenholzia zacatecae Ferris, 1922

NM Counties: Statewide.

Material Deposited: None.

Reference: Kim and others 1986.

Family Muridae: Subfamily Arvicolinae (Moles, Meadow Mice, Voles)

Genus Clethrionomys

Host: Clethrionomys gapperi (Vigor, 1830) . . . . . . . . Southern red-backed vole.

Siphonaptera:

1) Aetheca wagneri Baker, 1904

2) Amaradix euphorbae Rothschild, 1905

3) Catallagia decipiens Rothschild, 1915

4) Malaraeus telchinum Rothschild, 1905

5) Megabothris abantis Rothschild, 1905

6) Rhadinopsylla fraterna Baker, 1895

Species 1, 3, 4

NM Counties: Los Alamos (1, 3, 4); Rio Arriba (1, 3, 4); Sandoval (1, 3, 4); Santa Fe (1, 2, 3, 4, 6); Socorro (5).

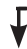

Species 5

Material Deposited: Flea MSB No.: (5) 998.

Reference: Fagerlund and others 2001; Haas and others 1973; Holdenried and Morlan 1956; Morlan 1955; Morlan and Prince 1954. 


\section{Host List-New Mexico Mammals}

\section{Class Mammalia}

Order Artiodactyla

Family Cervidae: (Deer and Elk)

Genus Odocoileus

Host: Odocoileus hemionus (Rafinesque, 1817) ............... Mule deer. Siphonaptera:

Ctenocephalides felis Bouche, 1835

NM Counties: Santa Fe.

Material Deposited: Flea MSB No.: 762.

Remarks/Observations: New state host record.

Reference: MSB Collection.

\section{Family Bovidae: (Antelopes, Cattle, Goats, and Sheep)}

Genus Capra

Host: Capra hircus Linnaeus, $1758 \ldots \ldots \ldots \ldots \ldots \ldots \ldots \ldots \ldots$. . . . . . . . . . . . . . . .

Anoplura:

Linognathus africanus Kellogg and Paine, 1911

NM Counties: Bernalillo.

Material Deposited: None.

Reference: Kim and others 1986.

Genus Ovis

Host: Ovis aries Linnaeus, $1758 \ldots \ldots \ldots \ldots \ldots \ldots \ldots$. . . . . . . . . . . . . .

Anoplura:

Linognathus africanus Kellogg and Paine, 1911

NM Counties: Bernalillo.

Material Deposited: None.

Reference: Kim and others 1986.

\section{Order Carnivora}

Family Canidae: (Dogs)

Genus Canis

Host: Canis familiaris Linnaeus, $1758 \ldots \ldots \ldots \ldots \ldots \ldots \ldots \ldots$. . . . . . . . . . . . . Siphonaptera:

1) Euhoplopsyllus glacialis Taschenberg, 1880

2) Ctenocephalides felis Bouche, 1835

3) Pulex irritans Linnaeus, 1758

4) Spilopsyllus inaequalis Baker, 1895 
Anoplura:

5) Linognathus setosus (von Olfers, 1816)

NM Counties: Bernalillo (2, 5); Sandoval $(1,3,4)$.

Material Deposited: Flea MSB No.: (2) 1567.

Reference: Haas and others 1973; MSB Collection.

Host: Canis latrans Say, $1823 \ldots \ldots \ldots \ldots \ldots \ldots \ldots$ Coyote. Siphonaptera:

1) Euhoplopsyllus glacialis Taschenberg, 1880

2) Spilopsyllus inaequalis Baker, 1895

Anoplura:

3) Linognathus setosus (von Olfers, 1816)

NM Counties: Bernalillo (3); Sandoval $(1,2)$.

Material Deposited: Flea MSB No.: (1) 859.

Reference: Haas and others 1973.

Genus Urocyon

Host: Urocyon cinereoargenteus (Schreber, 1775) .............. Gray fox. Siphonaptera:

1) Echidnophaga gallinaceus Westwood, 1875

2) Euhoplopsyllus affinis Baker, 1904

3) Foxella ignotus Baker, 1895

4) Pulex irritans Linnaeus, 1758

5) Pulex simulans Baker, 1895

6) Spilopsyllus inaequalis Baker, 1895

NM Counties: Bernalillo (1, 2, 3, 4, 5, 6); Socorro (1, 2, 3, 4, 5, 6).

Material Deposited: Flea MSB No.: (5) 830.

Reference: Patrick and Harrison 1995.

Genus Vulpes

Host: Vulpes macrotis macrotis Merriam, $1888 \ldots \ldots \ldots \ldots \ldots \ldots \ldots$ Kit fox. Siphonaptera:

1) Euhoplopsyllus affinis Baker, 1904

2) Foxella apachinus C. Fox, 1914

3) Orchopeas agilis Rothschild, 1905

4) Orchopeas caedens Jordan, 1925

5) Oropsylla montanus Baker, 1895

6) Pleochaetis exilis Jordan, 1937

7) Pulex irritans Linnaeus, 1758

8) Pulex simulans Baker, 1895

9) Spilopsyllus inaequalis Baker, 1895

10) Stenistomera alpina Baker, 1895

NM Counties: Chaves (1, 3, 4, 7, 8); DeBaca (6, 7); Eddy (4); Luna (7);

McKinley (2, 4, 5, 7, 10); San Juan (8, 9); Socorro (6); Torrance (7).

Material Deposited: None.

Reference: Harrison and others 2003.

Host: Vulpes velox velox (Say, 1823) . . . . . . . . . . . . . . . . Swift fox. Siphonaptera:

1) Echidnophaga gallinaceus Westwood, 1875

2) Euhoplopsyllus affinis Baker, 1904

3) Orchopeas agilis Rothschild, 1905

4) Orchopeas caedens Jordan, 1925 
5) Pulex irritans Linnaeus, 1758

6) Pulex simulans Baker, 1895

NM Counties: Chaves (5, 6); Dona Ana (3); Eddy (1); Lea (2, 3, 4, 5, 6);

McKinley (3); Roosevelt (5); Sandoval (3); Socorro (3); Union (5).

Material Deposited: Flea MSB No.: (1) 741; (5) 910; (6) 1048.

Reference: Harrison and others 2003.

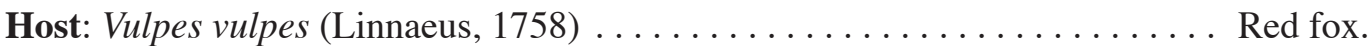
Siphonaptera:

1) Euhoplopsyllus affinis Baker, 1904

2) Pulex simulans Baker, 1895

3) Spilopsyllus inaequalis Baker, 1895

NM Counties: McKinley (3); Roosevelt (1, 2); San Juan (2).

Material Deposited: Flea MSB No.: (2) 916. Lice MSB No.: None accessioned.

Reference: Harrison and others 2003.

\section{Family Felidae: (Cats)}

Genus Felis

Host: Felis catus Linnaeus, $1758 \ldots \ldots \ldots \ldots \ldots \ldots \ldots \ldots \ldots$. . . . . . . . . . . Siphonaptera:

1) Echidnophaga gallinaceus Westwood, 1875

2) Euhoplopsyllus glacialis Taschenberg, 1880

3) Pulex irritans Linnaeus, 1758

4) Spilopsyllus inaequalis Baker, 1895

NM Counties: Bernalillo (4); Hidalgo (1); Sandoval (2, 3, 4).

Material Deposited: None.

Reference: Haas and others 1973; Jellison and Senger 1976.

Genus Lynx

Host: Lynx rufus (Schreber, 1777) . . . . . . . . . . . . . . . . . Bobcat. Siphonaptera:

1) Euhoplopsyllus glacialis Taschenberg, 1880

2) Foxella ignotus Baker, 1985

3) Spilopsyllus inaequalis Baker, 1895

4) Stenistomera alpina Baker, 1895

NM Counties: Sandoval (1, 2, 3, 4).

Material Deposited: None.

Reference: Haas and others 1973.

\section{Family Mephitidae: (Skunks)}

\section{Genus Mephitis}

Host: Mephitis sp.

Siphonaptera:

Echidnophaga gallinaceus Westwood, 1875

NM Counties: Santa Fe.

Material Deposited: Flea MSB No.: None accessioned.

Reference: Morlan 1955.

\section{Genus Spilogale}

Host: Spilogale gracilis Merriam, $1890 \ldots \ldots \ldots \ldots \ldots \ldots \ldots$ Western spotted skunk. 
Siphonaptera:

1) Anomiopsyllus nudata Baker, 1898

2) Hoplopsyllus anomalus Baker, 1904

3) Oropsylla montanus Baker, 1895

NM Counties: Santa Fe $(1,2,3)$.

Material Deposited: None.

Reference: Morlan 1955.

\section{Family Mustelidae: (Badgers and Weasels)}

Genus Mustela

Host: Mustela frenata Lichtenstein, $1831 \ldots \ldots \ldots \ldots \ldots$ Long-tailed weasel. Siphonaptera:

1) Foxella ignotus Baker, 1895

2) Megabothris abantis Rothschild, 1905

3) Thrassis pansus Jordan, 1925

4) Thrassis stanfordi Wagner, 1936

5) Stenistomera alpina Baker, 1895

NM Counties: Bernalillo (1); Rio Arriba (1, 3, 4); Sandoval (2).

Material Deposited: Flea MSB No.: (1) 1044.

Reference: Haas and others 1973; Link 1949; Traub and Hoff 1951.

\section{Family Procyonidae: (Raccoons and Relatives)}

Genus Bassariscus

Host: Bassariscus astutus (Lichtenstein, 1830) . . . . . . . . . . . . . . . Ringtail. Siphonaptera:

1) Aetheca wagneri Baker, 1904

2) Atyphloceras echis Jordan and Rothschild, 1915

3) Echidnophaga gallinaceus Westwood, 1875

4) Epitedia stanfordi Traub, 1944

5) Malaraeus sinomus Jordan, 1925

6) Megarthroglossus bisetis Jordan and Rothschild, 1915

7) Meringis arachis Jordan, 1929

8) Orchopeas sexdentatus neotomae Augustson, 1943

9) Oropsylla montanus Baker, 1895

10) Pulex simulans Baker, 1895

11) Rhadinopsylla goodi Hubbard, 1941

12) Stenistomera alpina Baker, 1895

13) Thrassis aridis Prince, 1944

NM Counties: Bernalillo (1, 2, 3, 4, 5, 6, 7, 8, 9, 10, 11, 12, 13); Sandoval (3).

Material Deposited: Flea MSB No.: (3) 1464; (8) 883.

Reference: Eads and others 1979, 1987; Fagerlund and others 2001;

Haas and others 1973; Jellison and Senger 1976.

\section{Order Chiroptera}

\section{Family Molossidae: (Molossid Bats)}

Genus Tadarida

Host: Tadarida brasiliensis I. Geoffroy, 1824 . . . . . . . . . . Brazilian free-tailed bat. 
Siphonaptera:

Sternopsylla texanus Fox, 1914

NM Counties: Eddy.

Material Deposited: None.

Reference: Jellison and Senger 1976.

\title{
Family Vespertilionidae: (Vesper Bats)
}

Genus Myotis

Host: Myotis thysanoides Miller, 1897.................. Fringed myotis.

Siphonaptera:

Myodopsylla nordina Traub and Hall, 1951

NM Counties: Bernalillo.

Material Deposited: None.

Reference: Traub and Hoff 1951.

Host: Myotis yumanensis $(\mathrm{H}$. Allen, 1864) . . . . . . . . . . . Yuma myotis. Siphonaptera:

Myodopsylla gentilis Jordan and Rothschild, 1921

NM Counties: Santa Fe.

Material Deposited: None.

Reference: Haas and others 1973.

\section{$\overline{\text { Order Insectivora }}$}

\section{Family Soricidae: (Shrews)}

\author{
Genus Sorex
}

Host: Sorex cinereus Kerr, 1792. . . . . . . . . . . . . . Cinereus shrew. Siphonaptera:

Corrodopsylla curvata Rothschild, 1915

NM Counties: Sandoval.

Material Deposited: Flea MSB No.: 1560.

Remarks/Observations: New state host record.

Reference: Haas and others 1973; MSB Collection.

Host: Sorex preblei Jackson, 1922 . . . . . . . . . . . . . . . . . . Preble's shrew. Siphonaptera:

Orchopeas agilis Rothschild, 1905

NM Counties: Sandoval.

Material Deposited: Flea MSB No.: 865.

Remarks/Observations: New state host record.

Reference: MSB Collection.

\section{Order Lagomorpha}

\section{Family Leporidae: (Hares)}

Genus Lepus

Host: Lepus californicus Gray, 1837 ............... Black-tailed jackrabbit. 


\section{Siphonaptera:}

1) Echidnophaga gallinaceus Westwood, 1875

2) Euhoplopsyllus affinis Baker, 1904

3) Euhoplopsyllus glacialis Taschenberg, 1880

4) Hoplopsyllus anomalus Baker, 1904

5) Pleochaetis exilis Jordan, 1937

6) Spilopsyllus inaequalis Baker, 1895

\section{Anoplura:}

7) Haemodipsus setoni Ewing, 1924

NM Counties: Bernalillo (2, 4); Chaves (3); Curry (3, 7); Dona Ana (2); Grant (1, 2); Santa Fe $(2,4,5)$; Torrance (2).

Material Deposited: None.

Reference: Fagerlund and others 2001; Holdenried and Morlan 1956; Jellison and Senger 1976; Kartman 1960; Kohls 1940; Morlan 1955; Pfaffenberger and Valencia 1988; Rodriguez 1977.

\section{Genus Sylvilagus}

Host: Sylvilagus audubonii (Baird, 1858) . . . . . . . . . . . . Desert cottontail.

\section{Siphonaptera:}

1) Aetheca wagneri Baker, 1904

2) Anomiopsyllus novomexicanensis Williams and Hoff, 1951

3) Echidnophaga gallinaceus Westwood, 1875

4) Euhoplopsyllus affinis Baker, 1904

5) Euhoplopsyllus glacialis Taschenberg, 1880

6) Megarthroglossus bisetis Jordan and Rothschild, 1915

7) Meringis bilsingi Eads and Menzies, 1949

8) Meringis dipodomys Kohls, 1938

9) Meringis nidi Williams and Hoff, 1951

10) Meringis rectus Morlan, 1953

11) Odontopsyllus dentatus Baker, 1904

12) Orchopeas sexdentatus Baker, 1904

13) Oropsylla hirsutus Baker, 1895

14) Polygenis gwynii C. Fox, 1914

15) Pulex irritans Linnaeus, 1758

16) Pulex simulans Baker, 1895

17) Rhadinopsylla fraterna Baker, 1895

18) Rhadinopsylla multidenticulatus Morlan and Prince, 1954

19) Spilopsyllus inaequalis Baker, 1895

20) Thrassis campestris Prince, 1944

21) Thrassis fotus Jordan, 1925

\section{Anoplura:}

22) Haemodipsus setoni Ewing, 1924

23) Hoplopleura hirsuta Ferris, 1916

NM Counties: Bernalillo (9); Catron (4); Chaves (1, 2, 3, 4, 5, 6, 7, 8, $9,10,12,14,15,16,17,18,19,20,21)$; Curry $(3,5,22)$; Grant $(3,4$, 8, 19); Lea (3, 5); Otero (4); Rio Arriba (4, 19); Roosevelt $(3,4,13$, 16, 23); Santa Fe (1, 4, 9, 10, 11, 12, 19).

Material Deposited: Flea MSB No.: (5) 723; (7) 792; (19) 748.

Reference: Clark and others 1971; Eads and others 1987; Graves and others 1974; Holdenried and Morlan 1956; Kartman 1960; Kohls 1940; Link 1949; Miller and others 1970; Morlan 1955; 
Pfaffenberger and Valencia 1988; Pfaffenberger and Wilson 1985;

Rail and others 1969; Rodriguez 1977.

Host: Sylvilagus floridanus (J.A. Allen, 1890). . . . . . . . . . . Eastern cottontail. Siphonaptera:

1) Euhoplopsyllus affinis Baker, 1904

NM Counties: Sandoval.

Material Deposited: Flea MSB No.: 749.

Reference: MSB Collection.

Host: Sylvilagus nuttalli (Bachman, 1837) . . . . . . . . . . Mountain cottontail. Siphonaptera:

1) Euhoplopsyllus affinis Baker, 1904

2) Megarthroglossus bisetis Jordan and Rothschild, 1915

3) Odontopsyllus dentatus Baker, 1904

4) Spilopsyllus inaequalis Baker, 1895

5) Stenistomera alpina Baker, 1895

NM Counties: Rio Arriba (1, 4); Sandoval (1, 2, 3, 5).

Material Deposited: None.

Reference: Haas and others 1973; Link 1949; Mendez and Haas 1973.

Host: Sylvilagus sp.

\section{Siphonaptera:}

1) Anomiopsyllus novomexicanensis Williams and Hoff, 1951

2) Echidnophaga gallinaceus Westwood, 1875

3) Euhoplopsyllus affinis Baker, 1904

4) Eumolpianus eumolpi Rothschild, 1905

5) Hoplopsyllus anomalus Baker, 1904

6) Megarthroglossus bisetis Jordan and Rothschild, 1915

7) Meringis bilsingi Eads and Menzies, 1949

8) Meringis dipodomys Kohls, 1938

9) Meringis nidi Williams and Hoff, 1951

10) Meringis rectus Morlan, 1953

11) Oropsylla montanus Baker, 1895

12) Polygenis gwyni Fox, 1914

13) Pulex irritans Linnaeus, 1758

14) Rhadinopsylla fraterna Baker, 1895

15) Thrassis fotus Jordan, 1925

NM Counties: Bernalillo (3, 4, 5); Chaves (1, 2, 3, 6, 7, 8, 9, 10, 12, 13,

14, 15); Sandoval (11).

Material Deposited: None.

Reference: Forcum and others 1969; Jellison and Senger 1976; Kohls 1940.

\section{Family Ochotonidae: (Pikas)}

\section{Genus Ochotona}

Host: Ochotona princeps (Richardson, 1828) .............. American pika. Siphonaptera:

1) Amphalius necopinus Jordan, 1925

2) Ctenophyllus armatus Wagner, 1901

3) Megabothris abantis Rothschild, 1905

4) Megarthroglossus bisetis Jordan and Rothschild, 1915 
5) Megarthroglossus divisus Baker, 1898

6) Phalacropsylla morlani Eads and Campos, 1982

7) Spilopsyllus inaequalis Baker, 1895

8) Stenistomera alpina Baker, 1895

NM Counties: Sandoval (4, 8); Santa Fe (1, 2, 3, 5, 6, 7, 8).

Material Deposited: None.

Reference: Haas and others 1973; Holdenried and Morlan 1956;

Mendez and Haas 1973; Morlan 1955.

\section{Order Perissodactyla}

\section{Family Equidae: (Equine)}

Genus Equus

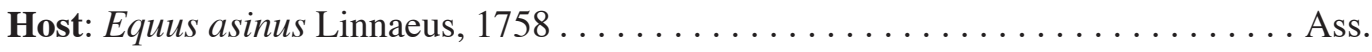
Anoplura;

1) Haematopinus asini Linnaeus, 1758

Mallophaga:

2) Werneckiella (Bovicola) equi (Denny, 1842)

NM Counties: Otero $(1,2)$.

Material Deposited: None.

Reference: Kim and others 1986.

Host: Equus caballus Linnaeus, $1758 \ldots \ldots \ldots \ldots \ldots \ldots \ldots \ldots$. . . . . . . . . . Anoplura:

1) Haematopinus asini Linnaeus, 1758

Mallophaga:

2) Werneckiella (Bovicola) equi (Denny, 1842)

NM Counties: Otero $(1,2)$.

Material Deposited: None.

Reference: Kim and others 1986.

\section{Order Primates}

\section{Family Hominidae: (Humans)}

\section{Genus Homo}

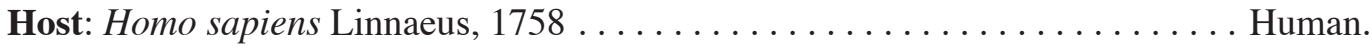
Siphonaptera:

1) Pulex irritans

Anoplura:

2) Pediculus humanus Linnaeus, 1758

3) Phthirus pubis (Linnaeus, 1758)

NM Counties: Bernalillo (2, 3); Los Alamos (1); Rio Arriba (1);

Sandoval (1); Santa Fe (1).

Material Deposited: None.

Reference: MSB Collection. 


\section{Order Rodentia}

\section{Family Geomyidae: (Pocket Gophers)}

Genus Thomomys

Host: Thomomys bottae (Eydoux and Gervais, 1836) . . . . . . B Botta's pocket gopher. Siphonaptera:

1) Foxella ignotus Baker, 1895

2) Spicata bottaceps Hubbard, 1943

NM Counties: Sandoval (1); Santa Fe $(1,2)$.

Material Deposited: None.

Reference: Fagerlund and others 2001; Haas and others 1973;

Holdenried and Morlan 1956; Morlan 1955.

Host: Thomomys talpoides (Richardson, 1828) . . . . . . . Northern pocket gopher. Siphonaptera:

1) Anomiopsyllus nudata Baker, 1898

2) Ctenophyllus armatus Wagner, 1901

3) Dactylopsylla neomexicana Prince, 1945

4) Eumolpianus eumolpi Rothschild, 1905

5) Foxella ignotus Baker, 1895

6) Megarthroglossus bisetis Jordan and Rothschild, 1915

7) Spicata rara Fox, 1940

NM Counties: San Miguel (3); Sandoval (5, 7); Santa Fe (1, 2, 4, 5, 6).

Material Deposited: None.

Reference: Haas 1973; Haas and others 1973; Holdenried and Morlan 1956; Morlan 1955; Prince 1945.

\section{Family Heteromyidae: (Kangaroo Rats, Pocket Mice)}

\section{Genus Chaetodipus}

Host: Chaetodipus hispidus (Baird, 1858) .............. Hispid pocket mouse. Anoplura:

Fahrenholzia zacatecae Ferris, 1922

NM Counties: Statewide.

Material Deposited: None.

Reference: Kim and others 1986.

Host: Chaetodipus intermedius Merriam, $1889 \ldots \ldots \ldots \ldots$. . . . Rock pocket mouse. Anoplura:

Fahrenholzia pinnata Kellogg and Ferris, 1915

NM Counties: Bernalillo, Luna, Otero, Roosevelt, Santa Fe, Socorro.

Material Deposited: None.

Reference: Kim and others 1986; Morlan and Hoff 1957; Pfaffenberger and deBruin 1986.

Genus Dipodomys

Host: Dipodomys merriami Mearns, $1890 \ldots \ldots \ldots \ldots \ldots$. . . . . Merriam's kangaroo rat. Siphonaptera:

1) Aetheca wagneri Baker, 1904

2) Anomiopsyllus novomexicanensis Williams and Hoff, 1951 
3) Anomiopsyllus hiemalis mexicanus Holland, 1965

4) Echidnophaga gallinaceus Westwood, 1875

5) Euhoplopsyllus affinis Taschenberg, 1880

6) Eumolpianus eumolpi Rothschild, 1905

7) Meringis altipecten Traub and Hoff, 1951

8) Meringis arachis Jordan, 1929

9) Meringis bilsingi Eads and Menzies, 1949

10) Meringis dipodomys Kohls, 1938

11) Meringis disparilis Eads, 1979

12) Meringis nidi Williams and Hoff, 1951

13) Meringis parkeri Jordan, 1937

14) Meringis rectus Morlan, 1953

15) Orchopeas leucopus Baker, 1904

16) Oropsylla montanus Baker, 1895

17) Rhadinopsylla fraterna Baker, 1895

18) Rhadinopsylla multidenticulatus Morlan and Prince, 1954

19) Thrassis campestris Prince, 1944

20) Thrassis fotus Jordan, 1925

\section{Anoplura:}

21) Fahrenholzia pinnata Kellogg and Ferris, 1915

22) Haematopinus asini Linnaeus, 1758

NM Counties: Chaves $(2,4,5,9,10,12,14,17,18,19,20)$; Colfax (3);

Dona Ana (7, 11); Eddy (11, 12); Hidalgo (8, 12); Luna (7, 11, 21); Otero $(9,12,14,21,22)$; Rio Arriba $(1,13)$; Santa Fe (15); Sierra (9, 13); Socorro $(5,6,15,16)$; Valencia (2).

Material Deposited: Flea MSB No.: (2) 794; (4) 714; (6) 724; (7) 1060; (8) 940; (9) 992; (10) 1028; (12) 1003; (13) 956; (15) 838; (16) $1011 ;$ (19) 875.

Reference: Barnes and others 1977; Eads 1978; Eads and others 1987; Graves and others 1974; Kim and others 1986; Link 1949; Miller and others 1970; Rail and others 1969; Traub and Hoff 1951.

Host: Dipodomys ordii Woodhouse, $1853 \ldots \ldots \ldots \ldots \ldots \ldots$ Ord's kangaroo rat. Siphonaptera:

1) Aetheca wagneri Baker, 1904

2) Anomiopsyllus novomexicanensis Williams and Hoff, 1951

3) Echidnophaga gallinaceus Westwood, 1875

4) Malaraeus sinomus Jordan, 1925

5) Meringis altipecten Traub and Hoff, 1951

6) Meringis arachis Jordan, 1929

7) Meringis bilsingi Eads and Menzies, 1949

8) Meringis dipodomys Kohls, 1938

9) Meringis disparilis Eads, 1979

10) Merinigs facilis Eads, 1979

11) Meringis nidi Williams and Hoff, 1951

12) Meringis parkeri Jordan, 1937

13) Meringis rectus Morlan, 1953

14) Orchopeas leucopus Baker, 1904

15) Peromyscopsylla adelpha Rothschild, 1915

16) Pleochaetis exilis Jordan, 1937

17) Rhadinopsylla multidenticulatus Morlan and Prince, 1954

18) Thrassis aridis Prince, 1944 
19) Thrassis bacchi consimilis Stark, 1957

20) Thrassis campestris Prince, 1944

21) Thrassis fotus Jordan, 1925

22) Thrassis pansus Jordan, 1925

\section{Anoplura:}

23) Fahrenholzia pinnata Kellogg and Ferris, 1915

24) Hoplopleura arboricola Kellogg and Ferris, 1915

25) Hoplopleura ferrisi Cook and Beer, 1959

26) Hoplopleura hesperomydis (Osborn, 1891)

27) Hoplopleura hirsuta Ferris, 1916

29) Neohaematopinus neotomae Ferris, 1941

30) Polyplax auricularis Kellogg and Ferris, 1915

\section{Mallophaga:}

31) Goniodes squamatus Emerson, 1950

NM Counties: Bernalillo (7, 31); Catron (12); Chaves (3, 7, 8, 10, 11, 13, 20); Dona Ana (2, 9); Grant (6); Hidalgo (18); Lea (8, 11, 12, 21); Luna (2, 7, 9); Roosevelt (7, 16, 18, 23, 27, 30); Sandoval (8, 12); Santa Fe (1, 11, 12, 13, 14, 15, 16, 17, 20, 22); Sierra (5, 6); Socorro (4); Torrance (13).

Material Deposited: Flea MSB No.: (3) 732; (4) 1027; (5) 957; (11) 1004; (12) 928; (18) 844; (19) 898.

Reference: Barnes and others 1977; Eads 1978; Eads and others 1987; Fagerlund and others 2001; Graves and others 1974; Haas and others 1973; Holdenried and Morlan 1956; Jellison and Senger 1976; Kartman 1960; Kim 1965; Kim and others 1986; Miller and others 1970; Morlan 1955; Morlan and Prince 1954; Pfaffenberger and deBruin 1986; Prince 1944; Rail and others 1969; Williams and Hoff 1951.

Host: Dipodomys spectabilis Merriam, $1890 \ldots \ldots \ldots \ldots$ Banner-tailed kangaroo rat. Siphonaptera:

1) Anomiopsyllus novomexicanensis Williams and Hoff, 1951

2) Echidnophaga gallinaceus Westwood, 1875

3) Euhoplopsyllus affinis Taschenberg, 1880

4) Megarthroglossus bisetis Jordan and Rothschild, 1915

5) Meringis altipecten Traub and Hoff, 1951

6) Meringis arachis Jordan, 1929

7) Meringis bilsingi Eads and Menzies, 1949

8) Meringis dipodomys Kohls, 1938

9) Meringis jamesoni Hubbard, 1943

10) Meringis nidi Williams and Hoff, 1951

11) Meringis parkeri Jordan, 1937

12) Meringis rectus Morlan, 1953

13) Orchopeas sexdentatus Baker, 1904

14) Pleochaetis exilis Jordan, 1937

15) Polygenis gwynii C. Fox, 1914

16) Pulex irritans Linnaeus, 1758

17) Pulex simulans Baker, 1895

18) Rhadinopsylla fraterna Baker, 1895

19) Rhadinopsylla multidenticulatus Morlan and Prince, 1954

20) Thrassis campestris Prince, 1944

21) Thrassis fotus Jordan, 1925

22) Thrassis pansus Jordan, 1925 
Anoplura:

23) Fahrenholzia pinnata Kellogg and Ferris, 1915

24) Hoplopleura acanthopus (Burmeister, 1839)

25) Hoplopleura arboricola Kellogg and Ferris, 1915

NM Counties: Bernalillo (10, 12, 25); Chaves (1, 2, 3, 4, 7, 8, 10, 12, 15, 16, 17, 18, 19, 20, 21); Dona Ana (6, 12); Lincoln (12); Luna (5, 6, 23); San Juan (12); Sandoval (12); Santa Fe (3, 6, 9, 10, 11, 12, 13, 19, 20, 22, 23, 24, 25); Sierra (6); Socorro (6); Valencia (12).

Material Deposited: Flea MSB No.: (2) 718; (5) 944; (6) 926; (8) 951; (10) 938.

Reference: Clark and others 1971; Eads and others 1987; Fagerlund and others 2001; Graves and others 1974; Holdenried and Morlan 1955, 1956; Jellison and Senger 1976; Kim and others 1986; Miller and others 1970; Morlan 1955; Morlan and Prince 1954; Rail and others 1969; Traub and Hoff 1951; Williams and Hoff 1951.

\section{Genus Perognathus}

Host: Perognathus flavescens Merriam, 1889 . . . . . . . . . Plains pocket mouse. Siphonaptera:

1) Meringis arachis Jordan, 1929

2) Thrassis pansus Jordan, 1925

NM Counties: Socorro $(1,2)$.

Material Deposited: Flea MSB No.: (1) 939; (3) 879.

Reference: MSB Collection.

Host: Perognathus flavus Baird, $1855 \ldots \ldots \ldots \ldots \ldots \ldots$. . . . . . . . . . . Siphonaptera:

1) Anomiopsyllus nudata Baker, 1898

2) Epitedia stanfordi Traub, 1944

3) Euhoplopsyllus affinis Baker, 1904

4) Meringis arachis Jordan, 1929

5) Meringis dipodomys Eads, 1979

6) Meringis facilis Eads, 1979

7) Meringis jamesoni Hubbard, 1943

8) Meringis nidi Williams and Hoff, 1951

9) Meringis parkeri Jordan, 1937

10) Meringis rectus Morlan, 1953

11) Orchopeas leucopus Baker, 1904

12) Oropsylla idahoensis Baker, 1904

Anoplura:

13) Fahrenholzia pinnata Kellogg and Ferris, 1915

NM Counties: Chaves (5, 6, 10); Santa Fe (1, 2, 3, 7, 8, 9, 10, 11, 12,

13); Socorro $(4,12)$; Union (7).

Material Deposited: Flea MSB No.: (4) 1000; (8) 931; (12) 1007.

Reference: Eads 1978; Eads and others 1987; Fagerlund and others 2001;

Graves and others 1974; Haas and others 1973; Holdenried and Morlan

1956; Kim and others 1986; Morlan 1955; Rail and others 1969.

\section{Family Muridae: Subfamily Arvicolinae (Moles, Meadow Mice, Voles)}

\section{Genus Clethrionomys}

Host: Clethrionomys gapperi (Vigor, 1830)............ Southern red-backed vole. 


\section{Siphonaptera:}

1) Aetheca wagneri Baker, 1904

2) Amaradix euphorbae Rothschild, 1905

3) Catallagia decipiens Rothschild, 1915

4) Malaraeus telchinum Rothschild, 1905

5) Megabothris abantis Rothschild, 1905

6) Rhadinopsylla fraterna Baker, 1895

NM Counties: Los Alamos (1, 3, 4); Rio Arriba (1, 3, 4); Sandoval (1, 3, 4); Santa Fe (1, 2, 3, 4, 6); Socorro (5).

Material Deposited: Flea MSB No.: (5) 998.

Reference: Fagerlund and others 2001; Haas and others 1973; Holdenried and Morlan 1956; Morlan 1955; Morlan and Prince 1954.

\section{Genus Microtus}

Host: Microtus longicaudus (Merriam, 1888) . . . . . . . . . . . Long-tailed vole. Siphonaptera:

1) Aetheca wagneri Baker, 1904

2) Catallagia decipiens Rothschild, 1915

3) Delotelis telegoni Rothschild, 1905

4) Eumolpianus eumolpi americanus Hubbard, 1950

5) Hystrichopsylla dippiei Rothschild, 1902

6) Malaraeus telchinum Rothschild, 1905

7) Megabothris abantis Rothschild, 1905

8) Megarthroglossus bisetis Jordan and Rothschild, 1915

9) Oropsylla idahoensis Baker, 1904

10) Peromyscopsylla adelpha Rothschild, 1915

11) Peromyscopsylla hamifer vigens Jordan, 1837

12) Peromyscopsylla selenis Rothschild, 1906

Anoplura:

13) Hoplopleura acanthopus (Burmeister, 1839)

14) Hoplopleura hesperomydis (Osborn, 1891)

NM Counties: Sandoval (1, 2, 5, 6, 7, 8, 9, 10, 11); Santa Fe (1, 2, 3, 4, $5,6,7,11,12,13,14)$.

Material Deposited: None.

Reference: Fagerlund and others 2001; Haas 1973; Haas and others 1973; Holdenried and Morlan 1956; Kim and others 1986; Mendez and Haas 1973; Morlan 1955.

Host: Microtus mexicanus (Saussure, 1861) ................. Mexican vole. Siphonaptera:

1) Ctenophthalmus pseudagyrtes Baker, 1904

2) Hystrichopsylla dippiei Rothschild, 1902

3) Plusaetis equatorius asetus Traub, 1950

NM Counties: Socorro $(1,2,3)$.

Material Deposited: Flea MSB No.: (1) 743; (2) 1017; (3) 947.

Reference: Fagerlund and others 2001; MSB Collection.

Host: Microtus montanus (Peale, 1848) . . . . . . . . . . . . . . Montane vole. Siphonaptera:

1) Aetheca wagneri Baker, 1904

2) Catallagia decipiens Rothschild, 1915 
3) Corrodopsylla curvata Rothschild, 1915

4) Dactylopsylla bluei Fox, 1909

5) Foxella ignotus Baker, 1895

6) Hystrichopsylla dippiei Rothschild, 1902

7) Malaraeus telchinum Rothschild, 1905

8) Megabothris abantis Rothschild, 1905

9) Peromyscopsylla hamifer vigens Jordan, 1937

10) Peromyscopsylla selenis Rothschild, 1906

11) Spicata rara Fox, 1940

NM Counties: San Miguel (4); Sandoval (1, 2, 3, 5, 6, 7, 8, 9, 10, 11).

Material Deposited: None.

Reference: Fagerlund and others 2001; Haas 1973; Haas and others 1973.

Host: Microtus pennsylvanicus (Ord, 1815).................. Meadow vole. Siphonaptera:

1) Megabothris megacolpus Jordan, 1929

2) Peromyscopsylla hamifer vigens Jordan, 1937

3) Peromyscopsylla selenis Rothschild, 1906

NM Counties: Colfax (1); San Miguel (2, 3); Sandoval (2, 3).

Material Deposited: None.

Reference: Johnson and Traub 1954.

Host: Microtus sp.

Siphonaptera:

1) Catallagia decipiens Rothschild, 1915

2) Megabothris abantis Rothschild, 1905

3) Meringis parkeri Jordan, 1937

4) Eumolpianus eumolpi Rothschild, 1905

NM Counties: Rio Arriba (1, 2, 3, 4).

Material Deposited: None.

Reference: Link 1949.

\section{Subfamily Murinae: (Old World Rats and Mice)}

Genus Mus

Host: Mus musculus Linnaeus, $1758 \ldots \ldots \ldots \ldots \ldots \ldots \ldots$. . . . . . . . . . . mouse. Siphonaptera:

Aetheca wagneri Baker, 1904

NM Counties: Sandoval.

Material Deposited: None.

Reference: Fagerlund and others 2001; Haas and others 1973.

\section{Genus Rattus}

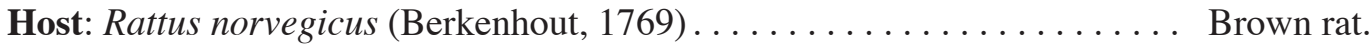
Siphonaptera:

1) Echidnophaga gallinaceus Westwood, 1875

2) Nosophyllus fasciatus Bosc, 1800

3) Xenopsylla cheopis Rothschild, 1903

NM Counties: Bernalillo (1, 2, 3); Chaves (1); Hidalgo (3); Valencia (2).

Material Deposited: None.

Reference: Becker 1947; Prince 1943. 


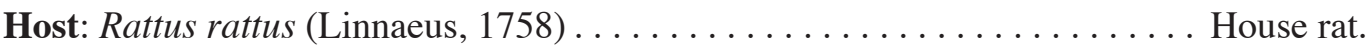
Siphonaptera:

Xenopsylla cheopis Rothschild, 1903

NM Counties: Hidalgo.

Material Deposited: None.

Reference: Becker 1947; Prince 1943.

\section{Subfamily Sigmodontinae: (New World Rats and Mice)}

\section{Genus Neotoma}

Host: Neotoma albigula Hartley, 1894. . . . . . . . . . . White-throated woodrat. Siphonaptera:

1) Aetheca wagneri Baker, 1904

2) Anomiopsyllus hiemalis Eads and Menzies, 1948

3) Anomiopsyllus hiemalis mexicanus Holland, 1965

4) Anomiopsyllus novomexicanensis Williams and Hoff, 1951

5) Anomiopsyllus nudata Baker, 1898

6) Atyphloceras echis Jordan and Rothschild, 1915

7) Echidnophaga gallinaceus Westwood, 1875

8) Epitedia stanfordi Traub, 1944

9) Hoplopsyllus anomalus Baker, 1904

10) Malaraeus sinomus Jordan, 1925

11) Megarthroglossus bisetis Jordan and Rothschild, 1915

12) Megarthroglossus divisus Baker, 1898

13) Megarthroglossus procus Jordan and Rothschild, 1915

14) Meringis altipecten Traub and Hoff, 1951

15) Meringis arachis Jordan, 1929

16) Meringis jamesoni Hubbard, 1943

17) Meringis nidi Williams and Hoff, 1951

18) Meringis parkeri Jordan, 1937

19) Meringis rectus Morlan, 1953

20) Orchopeas agilis Rothschild, 1905

21) Orchopeas leucopus Baker, 1904

22) Orchopeas sexdentatus Baker, 1904

23) Orchopeas sexdentatus neotomae Auguston, 1943

24) Oropsylla montanus Baker, 1895

25) Peromyscopsylla hesperomys Baker, 1904

26) Phalacropsylla hamata Tipton and Mendez, 1968

27) Pleochaetis exilis Jordan, 1937

28) Rhadinopsylla multidenticulatus Morlan and Prince, 1954

29) Rhadinopsylla sectilis Jordan and Rothschild, 1923

30) Stenistomera alpina Baker, 1895

31) Stenistomera macrodactyla Good, 1942

32) Thrassis bacchi consimilis Stark, 1957

33) Thrassis campestris Prince, 1944

34) Thrassis pansus Jordan, 1925

Anoplura:

35) Neohaematopinus neotomae Ferris, 1941

NM Counties: Bernalillo (3, 4, 11, 12, 22, 25, 26, 30, 35); Catron (3);

Chaves (2); Colfax (3); Dona Ana (4, 14, 17, 20); Eddy (2, 14); Grant

(6); Guadalupe (11); Hidalgo (4, 14, 15); Lea (2, 7, 22); Lincoln (3, 4, 12); Luna (4); McKinley (20); Otero (4, 12); Rio Arriba (1, 4, 7, 
22, 23); San Juan (4); Sandoval (11, 13, 29); Santa Fe (1, 3, 5, 6, 7, $8,9,10,11,12,16,17,18,19,21,22,23,24,28,30,31,33,34,35)$;

Sierra (4); Socorro (3, 4, 18, 20, 32); Valencia (4).

Material Deposited: Flea MSB No.: (3) 701; (4) 702; (5) 758; (7) 720; (12) 1008; (13) 1006; (15) 977; (20) 784; (21) 771; (23) 971; (32) 884.

Reference: Barnes and others 1977; Eads and Campos 1982; Eads and others 1987; Fagerlund and others 2001; Good 1942; Haas and others 1973; Holdenried and Morlan 1955, 1956; Kartman 1960; Kim and others 1986; Link 1949; Mendez 1956; Mendez and Haas 1973; Morlan 1955; Morlan and Prince 1954; Tipton and others 1979; Traub and Hoff 1951.

Host: Neotoma cinerea $($ Ord, 1815)................. Bushy-tailed woodrat. Siphonaptera:

1) Aetheca wagneri Baker, 1904

2) Amaradix vonfintelis Prince, 1959

3) Catallagia decipiens Rothschild, 1915

4) Hystrichopsylla dippiei Rothschild, 1902

5) Malaraeus telchinum Rothschild, 1905

6) Megarthroglossus bisetis Jordan and Rothschild, 1915

7) Megarthroglossus cavernicolus Mendez and Haas, 1972

8) Megarthroglossus divisus Baker, 1898

9) Orchopeas sexdentatus Baker, 1904

10) Orchopeas sexdentatus neotomae Auguston, 1943

11) Phalacropsylla allos Wagner, 1936

12) Rhadinopsylla fraterna Baker, 1895

13) Rhadinopsylla sectilis Jordan and Rothschild, 1923

14) Stenistomera alpina Baker, 1895

NM Counties: Colfax (6, 8); McKinley (6); Rio Arriba (9); Sandoval $(1,2,3,5,6,7,9,10,11,12,13,14)$; Santa Fe $(1,3,4,9,10,14)$.

Material Deposited: Flea MSB No.: (14) 814.

Reference: Eads and others 1987; Haas and others 1973; Holdenried and Morlan 1956; Link 1949; Mendez 1956; Mendez and Haas 1973; Morlan 1955; Tipton and others 1979.

Host: Neotoma lepida Thomas, $1893 \ldots \ldots \ldots \ldots \ldots \ldots \ldots$ Desert wood rat. Siphonaptera:

Anomiopsyllus novomexicanensis Williams and Hoff, 1951

NM Counties: Hidalgo.

Material Deposited: None.

Reference: Barnes and others 1977.

Host: Neotoma mexicana Baird, $1855 \ldots \ldots \ldots \ldots \ldots \ldots \ldots$ Mexican woodrat. Siphonaptera:

1) Aetheca wagneri Baker, 1904

2) Amaradix vonfintelis Prince, 1959

3) Anomiopsyllus hiemalis mexicanus Holland, 1965

4) Anomiopsyllus novomexicanensis Williams and Hoff, 1951

5) Anomiopsyllus nudata Baker, 1898

6) Catallagia decipiens Rothschild, 1915

7) Eumolpianus eumolpi Rothschild, 1905

8) Hystrichopsylla dippiei Rothschild, 1902 
9) Malaraeus sinomus Jordan, 1925

10) Malaraeus telchinum Rothschild, 1905

11) Megabothris abantis Rothschild, 1905

12) Megarthroglossus bisetis Jordan and Rothschild, 1915

13) Orchopeas sexdentatus Baker, 1904

14) Orchopeas sexdentatus neotomae Auguston, 1943

15) Phalacropsylla allos Wagner, 1936

16) Pleochaetis exilis Jordan, 1937

17) Stenistomera alpina Baker, 1895

Anoplura:

18) Neohaematopinus neotomae Ferris, 1941

NM Counties: Bernalillo (14, 18); Catron (3); McKinley (12); Rio Arriba (4); Sandoval (1, 2, 6, 8, 10, 11, 12, 13, 14, 15, 17); Santa Fe $(5,7,9,13,14,16,17,18)$; Taos (3).

Material Deposited: None.

Reference: Barnes and others 1977; Haas and others 1973; Holdenried and Morlan 1956; Kim and others 1986; Link 1949; Mendez and Haas 1973; Morlan 1955; Tipton and others 1979; Traub and Hoff 1951.

Host: Neotoma micropus Baird, $1855 \ldots \ldots \ldots \ldots \ldots$. . . . . . . . Siphonaptera:

1) Aetheca wagneri Baker, 1904

2) Anomiopsyllus novomexicanensis Williams and Hoff, 1951

3) Anomiopsyllus nudata Baker, 1898

4) Atyphloceras echis Jordan and Rothschild, 1915

5) Echidnophaga gallinaceus Westwood, 1875

6) Epitedia stanfordi Traub, 1944

7) Megarthroglossus bisetis Jordan and Rothschild, 1915

8) Megarthroglossus divisus Baker, 1898

9) Meringis bilsingi Eads and Menzies, 1949

10) Meringis dipodomys Kohls, 1938

11) Meringis facilis Eads, 1979

12) Meringis nidi Williams and Hoff, 1951

13) Meringis parkeri Jordan, 1937

14) Meringis rectus Morlan, 1953

15) Orchopeas leucopus Baker, 1904

16) Orchopeas sexdentatus Baker, 1904

17) Orchopeas sexdentatus neotomae Auguston, 1943

18) Peromyscopsylla draco Hopkins, 1951

19) Pleochaetis exilis Jordan, 1937

20) Polygenis gwynii (C. Fox, 1914)

21) Rhadinopsylla multidenticulatus Morlan and Prince, 1954

22) Thrassis campestris Prince, 1944

23) Thrassis fotus Jordan, 1925

24) Thrassis pansus Jordan, 1925

Anoplura:

25) Neohaematopinus neotomae Ferris, 1941

NM Counties: Bernalillo (2, 4, 6, 7, 13, 16); Chaves $(1,2,5,7,9,10$, 11, 12, 14, 16, 20, 21, 22, 23); Santa Fe (1, 3, 6, 7, 8, 12, 13, 14, 15, $16,17,18,19,21,22,24)$.

Material Deposited: None. 
Reference: Graves and others 1974; Holdenried and Morlan 1956; Jellison and Senger 1976; Kim and others 1986; Miller and others 1970; Morlan 1955; Morlan and Prince 1954; Williams and Hoff 1951.

Host: Neotoma sp.

\section{Siphonaptera:}

1) Anomiopsyllus novomexicanensis Williams and Hoff, 1951

2) Anomiopsyllus hiemalis mexicanus Holland, 1965

3) Atyphloceras echis Jordan and Rothschild, 1915

4) Echidnophaga gallinaceus Westwood, 1875

5) Euhoplopsyllus affinis Baker, 1904

6) Malaraeus telchinum Rothschild, 1905

7) Megarthroglossus bisetis Jordan and Rothschild, 1915

8) Meringis bilsingi Eads and Menzies, 1949

9) Meringis dipodomys Kohls, 1938

10) Meringis nidi Williams and Hoff, 1951

11) Meringis rectus Morlan, 1953

12) Orchopeas leucopus Baker, 1904

13) Orchopeas sexdentatus Baker, 1904

14) Polygenis gwynii C. Fox, 1914

15) Rhadinopsylla fraterna Baker, 1895

16) Thrassis campestris Prince, 1944

17) Thrassis fotus Jordan, 1925

\section{Anoplura:}

18) Linognathoides neotomae Ferris, 1942

NM Counties: Bernalillo (1, 3, 7, 18); Chaves (1, 3, 4, 5, 6, 7, 8, 9, 10, 11, 12, 13, 14, 15, 16, 17); Colfax (2); Otero (18); Lea (13); San Miguel (7); Santa Fe (7, 18); Valencia (2).

Material Deposited: None.

Reference: Barnes and others 1977; Jellison and Senger 1976; Jordan and Rothschild 1915; Kartman 1960; Kim and others 1986; Mendez 1956; Morlan 1955; Rail and others 1969.

\section{Genus Onychomys}

Host: Onychomys arenicola Mearns, $1896 \ldots \ldots \ldots$. . . Mearn's grasshopper mouse. Siphonaptera:

1) Meringis altipecten Traub and Hoff, 1951

2) Orchopeas caedens Jordan, 1925

NM Counties: Socorro $(1,2)$.

Material Deposited: Flea MSB No.: (1) 967; (2) 870.

Reference: MSB Collection.

Host: Onychomys leucogaster (Wied-Neuwied, 1841) . . Northern grasshopper mouse. Siphonaptera:

1) Aetheca wagneri Baker, 1904

2) Anomiopsyllus novomexicanensis Williams and Hoff, 1951

3) Echidnophaga gallinaceus Westwood, 1875

4) Epitedia stanfordi Traub, 1944

5) Malaraeus sinomus Jordan, 1925

6) Malaraeus telchinum Rothschild, 1905

7) Megarthroglossus bisetis Jordan and Rothschild, 1915

8) Megarthroglossus divisus Baker, 1898 
9) Meringis altipecten Traub and Hoff, 1951

10) Meringis arachis (Jordan, 1929)

11) Meringis bilsingi Eads and Menzies, 1949

12) Meringis dipodomys Kohls, 1938

13) Meringis disparilis Eads, 1979

14) Meringis facilis Eads, 1979

15) Meringis jamesoni Hubbard, 1943

16) Meringis nidi Williams and Hoff, 1951

17) Meringis parkeri Jordan, 1937

18) Meringis rectus Morlan, 1953

19) Orchopeas agilis Rothschild, 1905

20) Orchopeas leucopus Baker, 1904

21) Orchopeas sexdentatus Baker, 1904

22) Peromyscopsylla adelpha Rothschild, 1915

23) Peromyscopsylla draco Hopkins, 1951

24) Peromyscopsylla hesperomys Baker, 1904

25) Pleochaetis exilis Jordan, 1937

26) Rhadinopsylla multidenticulatus Morlan and Prince, 1954

27) Thrassis aridis Prince, 1944

28) Thrassis bacchi consimilis Stark, 1957

29) Thrassis campestris Prince, 1944

30) Thrassis fotus Jordan, 1925

31) Thrassis pansus Jordan, 1925

\section{Anoplura:}

32) Fahrenholzia pinnata Kellogg and Ferris, 1915

33) Hoplopleura hesperomydis (Osborn, 1891)

34) Hoplopleura hirsuta Ferris, 1916

35) Hoplopleura onychomydis Cook and Beer, 1959

36) Polyplax auricularis Kellogg and Ferris, 1915

NM Counties: Bernalillo (3, 14, 17, 31, 35); Chaves (2, 3, 7, 11, 12, 14, 16, 18, 23, 26, 27, 28, 29, 30); Colfax $(6,8,25)$; Dona Ana (2, 10, 11, 13); Eddy (30); Hidalgo (10, 27, 31); Lea (11, 21, 30); Luna (3, 9, 10, 13); Roosevelt (11, 20, 24, 25, 27, 30, 32, 33, 34, 36); San Juan (17, 25); Sandoval (11); Santa Fe (1, 4, 5, 15, 16, 17, 18, 19, 20, 21, 25, 26, 27, 28, 31); Socorro (9); Union (1, 5, 22, 23, 25); Valencia (14).

Material Deposited: Flea MSB No.: (5) 1016; (10) 994, 1032; (12) 1024; (20) 887; (25) 889; (28) 881.

Reference: Barnes and others 1977; Eads 1978; Eads and others 1987; Fagerlund and others 2001; Graves and others 1974; Jellison and Senger 1976; Kartman 1960; Kim and others 1986; Morlan 1955; Morlan and Prince 1954; Pfaffenberger and deBruin 1986; Prince 1944; Rail and others 1969; Thomas, 1988; Traub and Hoff 1951; Williams and Hoff 1951.

\section{Genus Peromyscus}

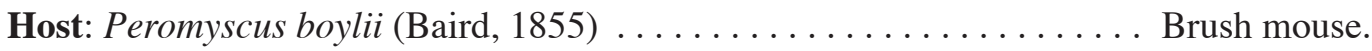
Siphonaptera:

1) Aetheca wagneri Baker, 1904

2) Anomiopsyllus novomexicanensis Williams and Hoff, 1951

3) Atyphloceras echis Jordan and Rothschild, 1915

4) Atyphloceras multidentatus C. Fox, 1909

5) Callistopsyllus terinus Rothschild, 1905 
6) Catallagia charlottensis Baker, 1898

7) Hoplopsyllus anomalus Baker, 1904

8) Malaraeus sinomus Jordan, 1925

9) Malaraeus telchinum Rothschild, 1905

10) Megarthroglossus bisetis Jordan and Rothschild, 1915

11) Megarthroglossus divisus Baker, 1898

12) Meringis altipecten Traub and Hoff, 1951

13) Orchopeas leucopus Baker, 1904

14) Peromyscopsylla adelpha Rothschild, 1915

15) Plusaetis equatorius asetus Traub, 1950

16) Rhadinopsylla sectilis Jordan and Rothschild, 1923

17) Stenoponia ponera Traub and Johnson, 1952

Anoplura:

18) Hoplopleura hesperomydis (Osborn, 1891)

NM Counties: Bernalillo (8, 11, 18); Grant (17); Lincoln (14); Los

Alamos (14); McKinley (11); Rio Arriba (10, 11, 14); Sandoval (1, 5, 9, 14, 16); Santa Fe (1, 3, 4, 6, 7, 8, 13, 14); Sierra (12); Socorro $(14,15)$; Valencia $(2,14)$.

Material Deposited: Flea MSB No.: (8) 933; (14) 834; (15) 831.

Reference: Barnes and others 1977; Eads and others 1987; Fagerlund and others 2001; Haas and others 1973; Holdenried and Morlan 1956; Kim 1965; Mendez 1956; Mendez and Haas 1973; Morlan 1955; Tipton and others 1979; Traub and Hoff 1951; Traub and Johnson 1952.

Host: Peromyscus eremicus (Baird, 1858) ............... Cactus mouse. Siphonaptera:

1) Anomiopsyllus hiemalis mexicanus Holland, 1965

2) Orchopeas leucopus Baker, 1904

Anoplura:

3) Hoplopleura ferrisi Cook and Beer, 1959

NM Counties: Colfax (1); Grant (3); Socorro (2).

Material Deposited: Flea MSB No.: (2) 840.

Reference: Barnes and others 1977; Kim 1965.

Host: Peromyscus leucopus (Rafinesque, 1818) ........... White-footed mouse. Siphonaptera:

1) Aetheca wagneri Baker, 1904

2) Anomiopsyllus novomexicanensis Williams and Hoff, 1951

3) Anomiopsyllus nudata Baker, 1898

4) Echidnophaga gallinaceus Westwood, 1875

5) Epitedia stanfordi Traub, 1944

6) Malaraeus sinomus Jordan, 1925

7) Megarthroglossus bisetis Jordan and Rothschild, 1915

8) Megarthroglossus divisus (Baker, 1898)

9) Meringis bilsingi Eads and Menzies, 1949

10) Meringis nidi Williams and Hoff, 1951

11) Meringis rectus Morlan, 1953

12) Orchopeas agilis Rothschild, 1905

13) Orchopeas caedens Jordan, 1925

14) Orchopeas leucopus Baker, 1904

15) Peromyscopsylla adelpha Rothschild, 1915 
16) Peromyscopsylla draco Hopkins, 1951

17) Peromyscopsylla hesperomys Baker, 1904

18) Phalacropsylla hamata Tipton and Mendez, 1968

19) Pleochaetis exilis Jordan, 1937

20) Rhadinopsylla fraterna Baker, 1895

21) Rhadinopsylla multidenticulatus Morlan and Prince, 1954

22) Rhadinopsylla sectilis Jordan and Rothschild, 1923

23) Thrassis campestris Prince, 1944

24) Thrasis fotus Jordan, 1925

25) Thrassis pansus Jordan, 1925

Anoplura:

26) Hoplopleura hesperomydis (Osborn, 1891)

NM Counties: Bernalillo (6, 7, 17, 18); Chaves $(1,2,7,9,10,11,14$, 16, 20, 21, 23, 24); Dona Ana (12); Sandoval (1, 5, 6, 12, 15, 22); Santa Fe (1, 3, 4, 5, 6, 8, 14, 15, 16, 19, 21, 23, 25); Socorro (12).

Material Deposited: Flea MSB No.: (4) 765; (6) 960;(12) 857; (13) 785.

Reference: Eads and Campos 1982; Graves and others 1974; Haas and others 1973; Holdenried and Morlan 1955, 1956; Kartman 1960; Kim and others 1986; Morlan 1955; Morlan and Prince 1954; Rail and others 1969; Traub and Hoff 1951.

Host: Peromyscus maniculatus (Wagner, 1845).................... Deer mouse. Siphonaptera:

1) Aetheca wagneri Baker, 1904

2) Amardix euphorbae Rothschild, 1905

3) Anomiopsyllus hiemalis mexicanus Holland, 1965

4) Anomiopsyllus novomexicanensis Williams and Hoff, 1951

5) Atyphloceras echis Jordan and Rothschild, 1915

6) Callistopsyllus terinus Rothschild, 1905

7) Catallagia decipiens Rothschild, 1915

8) Epitedia stanfordi Traub, 1944

9) Epitedia wnemanni Rothschild, 1904

10) Eumolpianus eumolpi Rothschild, 1905

11) Foxella ignotus Baker, 1895

12) Hystrichopsylla dippiei Rothschild, 1902

13) Malaraeus sinomus Jordan, 1925

14) Malaraeus telchinum Rothschild, 1905

15) Megabothris abantis Rothschild, 1905

16) Megarthroglossus bisetis Jordan and Rothschild, 1915

17) Megarthroglossus divisus Baker, 1898

18) Meringis altipecten Traub and Hoff, 1951

19) Meringis arachis Jordan, 1929

20) Meringis bilsingi Eads and Menzies, 1949

21) Meringis nidi Williams and Hoff, 1951

22) Meringis parkeri Jordan, 1937

23) Meringis rectus Morlan, 1953

24) Orchopeas leucopus Baker, 1904

25) Orchopeas sexdentatus Baker, 1904

26) Orchopeas sexdentatus neotomae Auguston, 1943

27) Peromyscopsylla adelpha Rothschild, 1915

28) Peromyscopsylla draco Hopkins, 1951

29) Peromyscopsylla hamifer vigens Jordan, 1937 
30) Peromyscopsylla hesperomys Baker, 1904

31) Peromyscopsylla selenis Rothschild, 1906

32) Phalacropsylla allos Wagner, 1936

33) Rhadinopsylla sectilis Jordan and Rothschild, 1923

34) Stenoponia americana Baker, 1899

35) Thrassis campestris Prince, 1944

36) Thrassis pansus Jordan, 1925

\section{Anoplura:}

37) Hoplopleura hesperomydis (Osborn, 1891)

38) Polyplax auricularis Kellogg and Ferris, 1915

NM Counties: Bernalillo (1, 4, 7, 13, 16, 17, 33, 37, 38); Dona Ana (20); Hidalgo (4, 5, 18, 19); Otero $(21,24)$; Rio Arriba $(1,7,9,10$, 14, 15, 24); San Juan (2); Sandoval (1, 7, 8, 11, 12, 13, 14, 15, 16, 22, 24, 26, 27, 29, 30, 31, 32, 33, 34, 37); Santa Fe (2, 3, 6, 7, 8, 10, 13, $14,17,21,22,23,24,25,27,28,33,35,36,37,38)$.

Material Deposited: Flea MSB No.: (1) 705; (2) 710; (8) 725; (13) 1001; (14) 987; (15) 982; (26) 852; (28) 854.

Reference: Barnes and others 1977; Eads and others 1987; Good 1942; Haas and others 1973; Holdenried and Morlan 1955, 1956; Kim and others 1986; Link 1949; Mendez 1956; Morlan 1955; Traub and Hoff 1951; Williams and Hoff 1951.

Host: Peromyscus nasutus (J.A. Allen, 1891) . . . . . . . . . . Northern rock mouse. Siphonaptera:

1) Aetheca wagneri Baker, 1904

2) Atyphloceras echis Jordan and Rothschild, 1915

3) Ceratophyllus vison Baker, 1904

4) Epitedia stanfordi Traub, 1944

5) Hystrichopsylla dippiei Rothschild, 1902

6) Malaraeus sinomus Jordan, 1925

7) Megarthroglossus bisetis Jordan and Rothschild, 1950

8) Peromyscopsylla adelpha Rothschild, 1915

9) Peromyscopsylla hesperomys Baker, 1904

10) Rhadinopsylla sectilis Jordan and Rothschild, 1923

11) Stenoponia americana Baker, 1899

Anoplura:

12) Hoplopleura hesperomydis (Osborn, 1891)

13) Polyplax auricularis Kellogg and Ferris, 1915

NM Counties: Bernalillo (1, 5, 6, 9, 12); Sandoval (11); Santa Fe (1, 2, $3,4,6,7,8,10,12,13)$.

Material Deposited: None.

Reference: Holdenried and Morlan 1956; Kim 1965; Kim and others 1986; Morlan 1955; Traub and Hoff 1951; Williams and Hoff 1951.

Host: Peromyscus sp.

Siphonaptera:

1) Aetheca wagneri Baker, 1904

2) Anomiopsyllus hiemalis mexicanus Holland, 1965

3) Atyphloceras echis Jordan and Rothschild, 1915

4) Callistopsyllus terinus Rothschild, 1905

5) Epitedia wenmanni Rothschild, 1904

6) Malaraeus sinomus Jordan, 1925 
7) Megarthroglossus bisetis Jordan and Rothschild, 1915

8) Megarthroglossus divisus Baker, 1898

NM Counties: Bernalillo (1, 3, 6, 7, 8); Rio Arriba (2); San Miguel (1, $2,4)$; Torrance (5).

Material Deposited: None.

Reference: Barnes and others 1977; Chapin 1919; Jellison and Senger 1976; Mendez 1956; Mendez and Haas 1973; Traub and Hoff 1951.

Host: Peromyscus truei (Shufeldt, 1885) . . . . . . . . . . . . Pinyon mouse. Siphonaptera:

1) Aetheca wagneri Baker, 1904

2) Amaradix euphorbae Rothschild, 1905

3) Anomiopsyllus hiemalis mexicanus Holland, 1965

4) Anomiopsyllus novomexicanensis Williams and Hoff, 1951

5) Anomiopsyllus nudata Baker, 1898

6) Atyphloceras echis Jordan and Rothschild, 1915

7) Callistopsyllus terinus Rothschild, 1905

8) Catallagia decipiens Rothschild, 1915

9) Echidnophaga gallinaceus Westwood, 1875

10) Epitedia stanfordi Traub, 1944

11) Epitedia wenmanni Rothschild, 1904

12) Euhoplopsyllus affinis Baker, 1904

13) Hystrichopsylla dippiei Rothschild, 1902

14) Malaraeus sinomus Jordan, 1925

15) Malaraeus telchinum Rothschild, 1905

16) Megabothris abantis Rothschild, 1905

17) Megarthroglossus bisetis Jordan and Rothschild, 1915

18) Megarthroglossus divisus Baker, 1898

19) Meringis nidi Williams and Hoff, 1951

20) Meringis rectus Morlan, 1953

21) Opisodasys keeni Baker, 1896

22) Orchopeas caedens Jordan, 1925

23) Orchopeas leucopus Baker, 1904

24) Orchopeas sexdentatus Baker, 1904

25) Orchopeas sexdentatus neotomae Auguston, 1943

26) Oropsylla montanus Baker, 1895

27) Peromyscopsylla adelpha Rothschild, 1915

28) Peromyscospylla draco Hopkins, 1951

29) Peromyscopsylla hesperomys Baker, 1904

30) Phalacropsylla allos Wagner, 1936

31) Pleochaetis exilis (Jordan, 1937)

32) Rhadinopsylla sectilis Jordan and Rothschild, 1923

33) Stenistomera alpina Baker, 1895

34) Stenistomera macrodactyla Good, 1942

35) Thrassis campestris Prince, 1944

Anoplura:

36) Hoplopleura hesperomydis (Osborn, 1891)

37) Polyplax auricularis Kellogg and Ferris, 1915

NM Counties: Bernalillo (4, 10, 14, 29, 33); Catron (3); Los Alamos

(17); McKinley (31); Rio Arriba (21); Sandoval (1, 6, 7, 8, 10, 13, 14, 15, 17, 18, 23, 25, 27, 28, 29, 32, 33, 34, 35); Santa Fe (1, 5, 6, 
7, 9, 10, 12, 14, 17, 18, 19, 20, 23, 24, 26, 27, 28); Socorro (16); Valencia (4).

Material Deposited: Flea MSB No.: (1) 703; (3) 707; (10) 739; (11) 737; (14) 932; (16) 998; (18) 997; (22) 827; (23) 770; (31) 901.

Reference: Barnes and others 1977; Fagerlund and others 2001; Haas and others 1973; Holdenried and Morlan 1955, 1956; Mendez and Haas 1973; Morlan 1955; Traub and Hoff 1951.

\section{Genus Reithrodontomys}

Host: Reithrodontomys megalotis (Baird, 1858) ......... Western harvest mouse. Siphonaptera:

1) Aetheca wagneri Baker, 1904

2) Echidnophaga gallinaceus Westwood, 1875

3) Epitedia stanfordi Traub, 1944

4) Malaraeus telchinum Rothschild, 1905

5) Megarthroglossus bisetis Jordan and Rothschild, 1915

6) Meringis bilsingi Eads and Menzies, 1949

7) Meringis nidi Williams and Hoff, 1951

8) Meringis rectus Morlan, 1953

9) Orchopeas caedens Jordan, 1925

10) Orchopeas leucopus Baker, 1904

11) Oropsylla montanus Baker, 1895

12) Peromyscopsylla adelpha Rothschild, 1915

13) Peromyscopsylla draco Hopkins, 1951

14) Rhadinopsylla sectilis Jordan and Rothschild, 1923

15) Thrassis fotus Jordan, 1925

16) Thrassis pansus Jordan, 1925

Anoplura:

17) Hoplopleura reithrodontomydis Ferris, 1951

NM Counties: Chaves (1, 5, 6, 8, 10, 13, 15); McKinley (9); Sandoval $(1,3,4,10,12,14,16)$; Santa Fe $(1,2,7,8,10,11,12,13)$; Socorro $(9,17)$; Taos (17).

Material Deposited: Flea MSB No.: (9) 858; (12) 893.

Reference: Graves and others 1974; Haas and others 1973;

Holdenried and Morlan 1955, 1956; Morlan 1955.

\section{Genus Sigmodon}

Host: Sigmodon hispidus Say and Ord, $1825 \ldots \ldots \ldots \ldots \ldots \ldots$. . . . . . . . . . . Siphonaptera:

1) Aetheca wagneri Baker, 1904

2) Anomiopsyllus novomexicanensis Williams and Hoff, 1951

3) Epitedia wenmanni Rothschild, 1904

4) Euhoplopsyllus affinis Baker, 1904

5) Euhoplopsyllus glacialis Taschenber, 1880

6) Megarthroglossus bisetis Jordan and Rothschild, 1915

7) Meringis bilsingi Eads and Menzies, 1949

8) Meringis nidi Williams and Hoff, 1951

9) Meringis rectus Morlan, 1953

10) Orchopeas leucopus Baker, 1904

11) Orchopeas sexdentatus Baker, 1904

12) Peromyscopsylla draco Hopkins, 1951

13) Pleochaetis exilis Jordan, 1937 
14) Polygenis gwyni (C. Fox, 1914)

15) Rhadinopsylla fraterna Baker, 1895

16) Rhadinopsylla multidenticulatus Morlan and Prince, 1954

17) Thrassis aridis Prince, 1944

18) Thrassis campestris Prince, 1944

19) Thrassis pansus Jordan, 1925

Anoplura:

20) Hoplopleura arizonensis Stojanovich and Pratt, 1961

21) Hoplopleura hirsuta Ferris, 1916

NM Counties: Chaves (1, 2, 3, 4, 6, 7, 8, 9, 10, 11, 12, 14, 15, 16, 18);

Roosevelt (5, 10, 13, 17, 21); Socorro (20).

Material Deposited: Flea MSB No.: (19) 864.

Reference: Clark and others 1971; Graves and others 1974; Miller and others 1970; Pfaffenberger and deBruin 1988; Rail and others 1969.

Host: Sigmodon ochrognathus Bailey, $1902 \ldots \ldots \ldots \ldots$. . . . . . . . . Siphonaptera:

Polygenis martinezbaezi Vargas, 1951

NM Counties: Hidalgo.

Material Deposited: None.

Reference: Haas and Wilson 1998.

\section{Family Sciuridae: (Squirrels, Prairie Dogs, and Chipmunks)}

Genus Ammospermophilus

Host: Ammospermophilus harrisii $\ldots \ldots \ldots \ldots \ldots \ldots$ Harris's antelope squirrel. (Audubon and Bachman, 1854)

Siphonaptera:

1) Thrassis pansus Jordan, 1925

Anoplura:

2) Enderleinellus suturalis Osborn, 1891

NM Counties: Bernalillo (2); Santa Fe (2); Socorro (1); Torrance (1).

Material Deposited: Flea MSB No.: (1) 813.

Reference: Jellison and Senger 1976; Kim and others 1986; MSB

Collection.

Host: Ammospermophilus leucurus (Merriam, 1889) .... White-tailed antelope squirrel. Siphonaptera:

Thrassis pansus Jordan, 1925

NM Counties: Socorro.

Material Deposited: Flea MSB No.: 818.

Remarks/Observations: New state host record.

Reference: MSB Collection.

Genus Cynomys

Host: Cynomys gunnisoni (Baird, 1855) ............ Gunnison's prairie dog. Siphonaptera:

1) Echidnophaga gallinaceus Westwood, 1875

2) Hoplopsyllus anomalus Baker, 1904

3) Meringis rectus Morlan, 1953

4) Oropsylla hirsutus Baker, 1895

5) Oropsylla idahoensis Baker, 1904 
6) Oropsylla labis Jordan and Rothschild, 1922

7) Oropsylla montanus Baker, 1895

8) Oropsylla tuberculatus cynomuris Jellison, 1939

9) Thrassi pansus Jordan, 1925

10) Thrassis stanfordi Wagner, 1936

\section{Anoplura:}

11) Enderleinellus suturalis Osborn, 1981

12) Linognathoides citellinus Ferris, 1942

13) Neohaematopinus citellinus Ferris, 1942

NM Counties: Bernalillo (4, 10); Colfax (5, 6, 8); Rio Arriba (4, 10); Sandoval (4, 5, 7, 8); Santa Fe (1, 2, 4, 8, 9, 11, 12, 13); Taos (4); Torrance (4).

Material Deposited: None.

Reference: Fagerlund and others 2001; Haas and others 1973; Holdenried and Morlan 1956; Hopkins and Rothschild 1953; Jellison and Senger 1976; Kim and others 1986; Link 1949; Morlan 1955; Morlan and Hoff 1957; O'Connor and Pfaffenberger 1987.

Host: Cynomys ludovicianus (Ord, 1815) . . . . . . . . . . Black-tailed prairie dog. Siphonaptera:

1) Oropsylla hirsutus Baker, 1895

2) Pulex simulans Baker, 1895

NM Counties: Chaves (1); Roosevelt $(1,2)$.

Material Deposited: None.

Reference: Clark and others 1971; Pfaffenberger and others 1984.

Host: Cynomys sp.

Siphonaptera:

1) Meringis parkeri Jordan, 1937

2) Oropsylla hirsutus Baker, 1895

NM Counties: Catron $(1,2)$.

Material Deposited: None.

Reference: Jellison and Senger 1976.

\section{Genus Marmota}

Host: Marmota flaviventris (Audubon and Bachman, 1841) ..... Yellow-bellied marmot. Siphonaptera:

1) Thrassis stanfordi Wagner, 1936

\section{Anoplura:}

2) Linognathoides marmotae (Ferris, 1923)

NM Counties: Rio Arriba (1); Santa Fe $(1,2)$.

Material Deposited: None.

Reference: Holdenried and Morlan 1956; Kim and others 1986; Link 1949; Morlan 1955.

\section{Genus Sciurus}

Host: Sciurus aberti Woodhouse, 1853 ...................... Abert's squirrel. Siphonaptera:

1) Anomiopsyllus martini Holland, 1965

2) Anomiopsyllus nudata Baker, 1898

3) Ceratophyllus vison Baker, 1904

4) Eumolpianus eumolpi americanus Hubbard, 1950 
5) Opisodasys robustus Jordan, 1925

6) Orchopeas caedens Jordan, 1925

7) Orchopeas sexdentatus neotomae Auguston, 1943

8) Tarsopsylla coloradensis Baker, 1895

NMCounties: Catron $(1,3,5,6)$; Sandoval $(2,4,5,7,8)$; Socorro $(5,6,7)$.

Material Deposited: Flea MSB Nos. respectively: (4) 1531; (5) 1534;

(7) 1551.

Reference: Barnes 1982; Barnes and others 1977; Haas and others 1973; Jellison 1939; Jordan 1925; Patrick and Wilson 1995.

Host: Sciurus arizonensis Coues, $1867 \ldots \ldots \ldots \ldots \ldots \ldots$.Arizona gray squirrel. Siphonaptera:

Orchopeas fulleri Traub, 1950

NM Counties: Catron.

Material Deposited: None.

Reference: Haas 1973.

\section{Genus Spermophilus}

Host: Spermophilus lateralis (Say, 1823) . . . . . . . Golden-mantled ground squirrel. Siphonaptera:

1) Catallagia decipiens Rothschild, 1915

2) Eumolpianus eumolpi Rothschild, 1905

3) Eumolpianus eumolpi americanus (Hubbard, 1950)

4) Hystrichopsylla dippiei Rothschild, 1902

5) Malaraeus telchinum Rothschild, 1905

6) Megabothris abantis Rothschild, 1905

7) Oropsylla hirsutus Baker, 1895

8) Oropsylla idahoensis Baker, 1904

9) Oropsylla montanus Baker, 1895

10) Oropsylla tuberculatus cynomuris Jellison, 1939

NM Counties: Rio Arriba (5, 8, 10); Sandoval (1, 3, 4, 6, 7, 8, 9); Santa

Fe $(2,8,9)$.

Material Deposited: None.

Reference: Haas and others 1973; Holdenried and Morlan 1956; Link 1949; Morlan 1955.

Host: Spermophilus spilosoma Bennett, $1833 \ldots \ldots \ldots \ldots$. . . . . . Siphonaptera:

1) Aetheca wagneri Baker, 1904

2) Echidnophaga gallinaceus Westwood, 1875

3) Euhoplopsyllus affinis Baker, 1904

4) Hoplopsyllus anomalus Baker, 1904

5) Meringis jamesoni Hubbard, 1943

6) Meringis nidi Williams and Hoff, 1951

7) Meringis parkeri Jordan, 1937

8) Meringis rectus Morlan, 1953

9) Orchopeas leucopus Baker, 1904

10) Thrassis bacchi consimilis Stark, 1957

11) Thrassis campestris Prince, 1944

12) Thrassis fotus Jordan, 1925

13) Thrassis pansus Jordan, 1925 


\section{Anoplura:}

14) Enderleinellus suturalis Osborn, 1891

15) Linognathoides spilosomae Stojanovich and Pratt, 1961

16) Neohaematopinus citellinus Ferris, 1942

17) Neohaematopinus spilosomae Stojanovich and Pratt, 1961

NM Counties: Bernalillo (2, 8, 13, 14, 15, 16, 17); Chaves (3, 11, 12);

Santa Fe $(1,2,4,5,6,7,8,9,13,14,16)$.

Material Deposited: Flea MSB No.: (2) 1599; (10) 868; (13) 823.

Reference: Graves and others 1974; Holdenried and Morlan 1956;

Jellison and Senger 1976; Kim et al, 1986; Rail and others 1969;

Williams and Hoff 1951.

Host: Spermophilus tridecemlineatus (Mitchill, 1821) . . .Thirteen-lined ground squirrel.

Siphonaptera:

Catallagia neweyi Holland and Loshbaugh, 1958

NM Counties: Colfax.

Material Deposited: None.

Reference: Fagerlund and others 2001.

Host: Spermophilus variegatus (Erxleben, 1777) .............. Rock squirrel. Siphonaptera:

1) Anomiopsyllus hiemalis mexicanus Holland, 1965

2) Echidnophaga gallinaceus Westwood, 1875

3) Hoplopsyllus anomalus Baker, 1904

4) Meringis rectus Morlan, 1953

5) Orchopeas caedens Jordan, 1925

6) Orchopeas leucopus Baker, 1904

7) Oropsylla hirsutus Baker, 1895

8) Oropsylla idahoensis Baker, 1904

9) Oropsylla montanus Baker, 1895

10) Thrassis fotus Jordan, 1925

Anoplura:

11) Linognathoides laeviusculus (Grube, 1851)

12) Neohaematopinus citellinus Ferris, 1842

NM Counties: Bernalillo (2, 3, 7, 11); Catron (1); Otero (5, 9); Rio Arriba (7); Sandoval (2, 3, 8); Santa Fe (2, 3, 4, 7, 10, 11, 12).

Material Deposited: Flea MSB No.: (3) 733; (6) 930.

Reference: Barnes and others 1977; Haas and others 1973; Holdenried and Morlan 1955, 1956; Jellison and Senger 1976; Kim and others 1986; Link 1949; Morlan 1955; Williams and Hoff 1951.

\section{Genus Tamias}

Host: Tamias dorsalis Baird, $1855 \ldots \ldots \ldots \ldots \ldots \ldots \ldots \ldots$. . . . . . . . . chipmunk. Siphonaptera:

Eumolpianus eumolpi americanus Hubbard, 1950

NM Counties: McKinley.

Material Deposited: Flea MSB No.: 717.

Reference: MSB Collection.

Host: Tamias minimus Bachman, $1839 \ldots \ldots \ldots \ldots \ldots \ldots$. . . . . . . . . chipmunk. Siphonaptera:

1) Aetheca wagneri Baker, 1904

2) Anomiopsyllus nudata Baker, 1898

3) Ceratophyllus vison Baker, 1904 
4) Eumolpianus eumolpi Rothschild, 1905

5) Eumolpianus eumolpi americanus (Hubbard, 1950)

6) Hystrichopsylla dippiei Rothschild, 1902

7) Megarthroglossus wilsoni Mendez and Haas, 1973

8) Oropsylla idahoensis Baker, 1905

NM Counties: Colfax (7); Sandoval (1, 2, 3, 5, 6, 8); Santa Fe (4).

Material Deposited: None.

Reference: Fagerlund and others 2001; Haas and others 1973;

Holdenried and Morlan 1956; Morlan 1955; Tipton and others 1979.

Host: Tamias quadrivittatus (Say, 1823) . .............. Colorado chipmunk. Siphonaptera:

1) Aetheca wagneri Baker, 1904

2) Anomiopsyllus nudata Baker, 1898

3) Catallagia decipiens Rothschild, 1915

4) Epitedia stanfordi Traub, 1944

5) Eumolpianus eumolpi Rothschild, 1905

6) Eumolpianus eumolpi americanus (Hubbard, 1950)

7) Hystrichopsylla dippiei Rothschild, 1902

8) Orchopeas leucopus Baker, 1904

9) Oropsylla idahoensis Baker, 1904

10) Peromyscopsylla adelpha Rothschild, 1915

11) Stenistomera alpina Baker, 1895

Anoplura:

12) Hoplopleura arboricola Kellog and Ferris, 1915

13) Neohaemotopinus pacificus Kellogg and Ferris, 1915

NM Counties: Bernalillo (5, 7, 11, 12); Rio Arriba (5, 8); Sandoval (1, 2, 3, 4, 6, 7); Santa Fe (1, 5, 7, 9, 10, 11, 13); Socorro (12).

Material Deposited: None.

Reference: Fagerlund and others 2001; Haas and others 1973; Holdenried and Morlan 1955, 1956; Kim and others 1986; Link 1949; Morlan 1955; Traub and Hoff 1951.

Host: Tamias sp.

Siphonaptera:

1) Eumolpianus eumolpi Rothschild, 1905

2) Eumolpi eumolpi americanus Hubbard, 1950

Anoplura:

3) Hoplopleura arboricola Kellogg and Ferris, 1915

4) Linognathoides pacificus Kellogg and Ferris, 1915

NM Counties: Bernalillo (3); Otero (1, 2); Santa Fe (4).

Material Deposited: Flea MSB No.: (1) 747.

Reference: Jellison and Senger 1976; Kim and others 1986.

\section{Genus Tamiasciuris}

Host: Tamiasciurus hudsonicus (Erxleben, 1777) ................ Red squirrel. Siphonaptera:

1) Aetheca wagneri Baker, 1904

2) Amaradix bitterootensis Dunn, 1923

3) Catallagia decipiens Rothschild, 1915

4) Ceratophyllus vison Baker, 1904

5) Eumolpianus eumolpi Rothschild, 1915 
6) Eumolpianus eumolpi americanus (Hubbard, 1950)

7) Hystrichopsylla dippiei Rothschild, 1902

8) Malaraeus telchinum Rothschild, 1905

9) Megarthroglossus bisetis Jordan and Rothschild, 1915

10) Megarthroglossus divisus Baker, 1898

11) Opisodasys robustus Jordan, 1925

12) Orchopeas caedens Jordan, 1925

13) Orchopeas leucopus Baker, 1904

14) Orchopeas sexdentatus Baker, 1904

15) Orchopeas sexdentatus neotomae Auguston, 1943

16) Oropsylla idahoensis Baker, 1904

17) Tarsopsylla coloradensis Baker, 1895

NM Counties: Catron (4, 11, 12, 16); Los Alamos (4); Sandoval (1, 2, $3,4,6,7,8,9,12,13,14,15)$; Santa Fe $(4,5,12,17)$; Taos $(4,11)$; Valencia (9).

Material Deposited: Flea MSB No.: (2) 1529; (3) 1530; (11) 1538; (12) 1543.

Reference: Haas 1972; Haas and others 1973; Holdenried and Morlan 1956; Mendez and Haas 1973; Morlan 1955; Patrick and Wilson, 1995. 


\section{References}

Barnes, A. M.; Tipton, V. J.; Wilde, J. A. 1977. The subfamily Anomiopsyllinae (Hystrichopsyllidae: Siphonaptera). I. A revision of the genus Anomiopsyllus Baker. Great Basin Naturalist. 37(2): 138-206.

Barnes, A. M. 1982. Surveillance and control of bubonic plague in the United States. Symposia of the Zoological Society of London. 50: 237-270.

Becker, E. R. 1947. Distribution of the tropical rat flea (Xenopsylla cheopis) in the interior of the United States. Iowa Academy of Science. 54: 297-299.

Bermudez, F. C.; Stuart, J. N.; Frey, J. K.; Valdez, R. 1995. Distribution and status of the Virginia opossum (Didelphis virginiana) in New Mexico. Southwestern Naturalist. 40(3): 336-340.

Brooks, D. R. 1993. Critical Comment: Extending the symbiotype concept to host voucher specimens. Journal of Parasitology. 79: 631-633.

Brown, T. Undated. A guide to the names of animals known to be infected in New Mexico. Unpublished list. New Mexico Environmental Division, Vector Control. 1 p.

Busvine, J. R. 1978. Evidence from double infestations for the specific status of human head lice and body lice (Anoplura). Systematic Entomology. 3: 1-8.

Centers for Disease Control and Prevention. 2003. Imported plague-New York City, 2002. Morbidity and Mortality Weekly Report. 52: 725-728.

Chapin, E. A. 1919. New species of North American Siphonaptera. Bulletin of the Brooklyn Entomological Society. 14(2): 49-62.

Clark, P. H.; Cole, M. M.; Forcum, D. L.; Wheeler, J. R.; Wheeler, K.; Miller, B. E. 1971. Preliminary evaluation of three systemic insecticides in baits for control of fleas of wild rats and rabbits. Journal of Economic Entomology. 64: 1190-1193.

Dryden, M. W.; Broce, A. B. 1993. Development of a trap for collecting newly emerged Ctenocephalides felis (Siphonaptera: Pulicidae) in homes. Journal of Medical Entomology. 30: 901-906.

Eads, R. E. 1978. Two new species of fleas of the genus Meringis (Siphonaptera: Hystrichopsyllidae). Great Basin Naturalist. 38(4): 447-455.

Eads, R. E.; Campos, E. G. 1982. Description of a new Phalacropsylla and notes on $P$. allos (Siphonaptera: Hystrichopsyllidae). Great Basin Naturalist. 42(2): 241245.

Eads, R. E.; Campos, E. G.; Barnes, A. M. 1979. New records for several flea (Siphonaptera) species in the United States, with observations on species parasitizing carnivores in the Rocky Mountain region. Proceedings of the Entomological Society of Washington. 81(1): 38-42.

Eads, R. E.; Campos, E. G.; Maupin, G. O. 1987. A review of the genus Meringis (Siphonaptera: Hystrichopsyllidae). Journal of Medical Entomology. 24(4): 467-476.

Fagerlund, R.; Ford, P. L.; Brown, T.; Polechla, P. J., Jr. 2001. New records for fleas (Siphonaptera) from New Mexico with notes on plague-carrying species. Southwestern Naturalist. 46: 94-95.

Forcum, D. L.; Rael, C. D.; Wheeler, J. R.; Miller, B. E. 1969. Abundance of cottontails and their fleas at Red Bluff
Ranch, New Mexico. Journal of Wildlife Management. 33(2): 422-424.

Frey, J. K.; Yates, T. L. 1996. Mammalian diversity in New Mexico. New Mexico Journal of Science. 36: 4-37.

Frey, J. K.; Yates, T. L.; Duszynski, D. W.; Gannon, W. L.; Gardner, S. L. 1992. Designation and curatorial management of type host specimens (symbiotypes) for new parasite species. Journal of Parasitology. 78: 930932.

Gardner, S. L. 1996. Field parasitology techniques for use with mammals. In: Wilson, D. E., Cole, F. R., Nichols, J. D., Rudran, R., Foster, M. S., eds. Measuring and monitoring biological diversity: standard methods for mammals. Washington, DC: Smithsonian Institution Press. p. 291-298.

Good, N. E. 1942. Key to the males of the genus Atyphloceras with a description of the male of

Atyphloceras echis (Siphonaptera). Pan-Pacific Entomologist. 28(2): 87-90.

Graves, G. N.; Bennett, W. C.; Wheeler, J. R.; Miller, B. E.; Forcum, D. L. 1974. Sylvatic plague studies in southeast New Mexico. Journal of Medical Entomology. 11(4): 488498.

Haas, G. E. 1972. Partial castration in Monopsyllus vison (Baker) (Siphonaptera). Entomological News. 83: 275278.

Haas, G. E. 1973. Morphological notes on some Siphonaptera (Leptopsyllidae and Ceratophyllidae) of New Mexico. America Midland Naturalist. 90(1): 246-252.

Haas, G.; Wilson, N. 1998. Polygenis martinezbaezi (Siphonaptera: Rhopalopsyllidae) reared from a rodent nest found in the Peloncillo Mountains of southwestern New Mexico. Journal of Medical Entomology. 35(4): 431432.

Haas, G.; Martin, R. P.; Swickard, M.; Miller, B. E. 1973. Siphonaptera-mammal relationships in northcentral New Mexico. Journal of Medical Entomology. 10(3): 281-289.

Harrison, R. L.; Patrick, M. J.; Schmitt, C. G. 2003. Foxes, fleas, and plague in New Mexico. Southwestern Naturalist. In press.

Holdenried, R.; Morlan, H. B. 1955. Plague-infected fleas from northern New Mexico wild rodents. Journal of Infectious Diseases. 96: 133-137.

Holdenried, R.; Morlan, H. B. 1956. A field study of wild mammals and fleas of Santa Fe County, New Mexico. American Midland Naturalist. 55(2): 369-381.

Hopkins, G. H. E.; Rothschild, M. 1953. An illustrated catalogue of the Rothschild collection of fleas (Siphonaptera) in the British Museum (Natural History), Vol. 1 Tungidae and Pulicidae. British Museum of Natural History. $361 \mathrm{p}$.

Hubbard, J. P.; Schmitt, C. G 1984. The black-footed ferret in New Mexico. Final report to the Bureau of Land Management, Santa Fe, NM. 118 p.

Jellison, W. J. 1939. Opisodasys Jordan 1933, a genus of Siphonaptera. Journal of Parasitology. 25: 413-420.

Jellison, W. J.; Senger, S. M. 1976. Fleas of western North America except Montana in the Rocky Mountain laboratory collection. Western Washington State College, Bellingham, WA. p. 55-136.

Johnson, P. T.; Traub, R. 1954. Revision of the flea genus Peromyscopyslla. Smithsonian Miscellaneous Collections. 123(4): 1-68. 
Jordan, K. 1925. New Siphonaptera. Novitates Zoologicae. 32: $96-112$.

Jordan, K.; Rothschild, N. C. 1915. Contribution to our knowledge of American Siphonaptera. Ectoparasites. 1: 45-60.

Kartman, L. 1960. The role of rabbits in sylvatic plague epidemiology, with special attention to human cases in New Mexico and use of the fluorescent antibody technique for detection of Pasteurella pestis in field specimens. Zoonoses Research. 1: 1-27.

Kim, K. C. 1965. A review of the Hoplopleura hesperomydis complex (Anoplura: Hoplopleuridae). Journal of Parasitology. 51(5): 871-887.

Kim, K. C. 1985. Coevolution of parasitic arthropods and mammals. John Wiley and Sons, New York, NY. 800 p.

Kim, K. C.; Pratt, H. D.; Stojanovich, C. J. 1986. The sucking lice of North America, an illustrated manual for identification. Pennsylvania State University Press. 241 p.

Kohls, G. M. 1940. Siphonaptera, a study of the species infesting wild hares and rabbits of North America north of Mexico. United States Public Health Service, National Institutes of Health Bulletin. 175: 1-34.

Laney, L. H. 1950. Annual report, rodent control, New Mexico District. U.S. District. U.S. Fish and Wildlife Service, and New Mexico College of Agriculture and Mechanical Arts, Extension Service. 10 p.

Lechleitner, R. R.; Tileson, J. V.; Kartman, L. 1962. Die-off of a Gunnison's prairie dog colony in central Colorado. 1. Ecological observations and description of the epizootic. Zoonoses Research. 1: 185-199.

Link, V. B. 1949. Plague among wild rodents in Rio Arriba County, New Mexico. American Journal of Tropical Medicine. 29: 493-500.

Marquardt, W. C.; Demaree, R. S.; Grieve, R. B. 2000. Parasitology and vector biology. San Diego, CA: Harcourt Academic Press. 697 p.

Mendez, E. 1956. A revision of the genus Megarthroglossus Jordan and Rothschild 1915 (Siphonaptera: Hystrichopsyllidae). University of California Publications in Entomology. 11: 159-192.

Mendez, E.; Haas, G. E. 1973. A new flea of the genus Megarthroglossus Jordan and Rothschild from New Mexico (Siphonaptera: Hystrichopsyllidae: Anomiopsyllinae). Journal of Medical Entomology. 9(4): 285-288.

Miller, B. E.; Forcum, D. L.; Weeks, K. W.; Wheeler, J. R.; Rail, C. D. 1970. An evaluation of insecticides for flea control on wild mammals. Journal of Medical Entomology. 7: 697-702.

Morlan, H. B. 1955. Mammal fleas of Santa Fe County, New Mexico. Texas Reports on Biology and Medicine. 13(1): 93-125.

Morlan, H. B.; Hoff, C. C. 1957. Notes on some Anoplura from New Mexico and Mexico. Journal of Parasitology. 43(3): 347-351.

Morlan, H. B.; Prince, F. M. 1954. Notes of the subfamily Rhadinopsyllinae Wagner 1930 (Siphonaptera: Hystrichopsyllidae) and description of a new species, Rhadinopsylla multidenticulatus. Texas Reports on Biology and Medicine. 12: 1037-1046.

OConnor, B.; Pfaffenberger, G. S. 1987. Systematics and evolution of the genus Paraceroglyphus and related taxa (Acari: Acaridae) associated with fleas (Insecta: Siphonaptera). Journal of Parasitology. 73(6): 1189-1197.
Patrick, M. J.; Harrison, R. L. 1995. Fleas on gray foxes in New Mexico. Journal of Medical Entomology. 32: 201204.

Patrick, M. J.; Wilson, W. D. 1995. Parasites of Aberts squirrel (Sciurus aberti) and red squirrel (Tamiasciurus hudsonicus) of New Mexico. Journal of Parasitology. 81(2): 321-324.

Pfaffenberger, G. S.; deBruin, D. 1986. Ectoparasitic overlap between sympatric Dipodomys ordii and Onychomys leucogaster (Rodentia) in eastern New Mexico, USA. Journal of Medical Entomology. 23(2): 201-207.

Pfaffenberger, G. S.; deBruin, D. 1988. Parasites of the hispid cotton rat, Sigmodon hispidus (Cricetinae), and population biology of the cotton rat louse, Hoplopleura hirsuta (Hoplopleuridae: Anoplura), in eastern New Mexico, including an annotated host-parasite bibliography. Texas Journal of Science. 40(4): 369-399.

Pfaffenberger, G. S.; Valencia V. B. 1988. Ectoparasites of sympatric cottontails (Sylvilagus audubonii Nelson) and jack rabbits (Lepus californicus Mearns) from the high plains of eastern New Mexico. Journal of Parasitology. 74(5): 842-846.

Pfaffenberger, G. S.; Wilson, C. 1985. Ectoparasites of vertebrates cohabiting black-tailed prairie dog towns in eastern New Mexico. Journal of Wildlife Diseases. 21: 6972.

Pfaffenberger, G. S.; Nygren, B.; deBruin, D.; Wilson, C. 1984. Parasites of the black-tailed prairie dog (Cynomys ludovicianus) from eastern New Mexico. Proceedings of the Helminthological Society of Washington. 51(2): 241244.

Prince, F. M. 1943. Species of fleas on rats collected in states west of the $102^{\text {nd }}$ meridian and their relation to the dissemination of plague. Public Health Reports. 58: 700708.

Prince, F. M. 1944. Descriptions of three new species of Thrassis Jordan and the females of $T$. bacchi Rothschild and T. pansus Jordan. Pan-Pacific Entomologist. 20(1): 1319.

Prince, F. M. 1945. Descriptions of three new species of Dactyopsylla Jordan and one new subspecies of Foxella Wagner, with records of other species in the genera (Siphonaptera). Canadian Entomologist. 77: 15-20.

Rail, C. D.; Forcum, D. L.; Wheeler, J. R.; Miller, B. E. 1969. Wild mammals and fleas of Red Bluff Ranch, New Mexico. Journal of Medical Entomology. 6(1): 92-94.

Roberts, L. S.; Janovy, J., Jr. 2000. Foundations of parasitology (6 $6^{\text {th }}$ ed.). Boston, MA: McGraw-Hill, p. 539-549, 559-569.

Rodriguez, P. H. 1977. A survey of ectoparasites of hares and rabbits in Grant County, New Mexico. Texas Journal of Science. 28: 358.

Rollag, O. J.; Skeels, M. R.; Nims, L. J.; Thilsted, J. P.; Mann, J. M. 1981. Feline plague in New Mexico: report of five cases. AmericanVeterinary Medical Association. 179: 1381-1383.

Service, M. W. 2000. Medical entomology for students $\left(2^{\text {nd }}\right.$ ed.). Cambridge, UK: Cambridge University Press. p. 167192.

Thomas, R. E. 1988. A review of flea collection records from Onychomys leucogaster with observations on the role of grasshopper mice in the epizoology of wild rodent plague. Great Basin Naturalist. 48: 83-95.

Thomas, R. E. 1996. Fleas and the agents they transmit. In: Beaty, B. J., Marquardt, W. C., eds. The biology of disease 
vectors. Niwot, CO: University Press of Colorado. p. 146159.

Tipton, V. J.; Stark, H. E.; Wildie, J. A. 1979. Anomiopsyllinae (Siphonaptera: Hystrichopsyllinae), II. The genera Callistopsyllus, Conorhinopsylla, Megarthroglossus, and Stenistomera. Great Basin Naturalist. 39(4): 351-418.

Traub, R.; Hoff, C. C. 1951. Records and descriptions of fleas from New Mexico (Siphonaptera). American Museum Novitates. 1530: 1-23.

Traub, R.; Johnson, P. T. 1952. Kohlsia whartoni and Stenoponia ponera, new species of fleas from North America. Journal of Parasitology. 38: 6-18.

Whitaker, J. O. 1982. Ectoparasites of mammals of Indiana. Indianapolis, IN: Indiana Academy of Sciences. 240 p.

Williams, L. A.; Hoff, C. C. 1951. Fleas from the upper sonoran zone near Albuquerque, New Mexico. Proceedings of the United States National Museum. 101(3278): 305-313.

Zinsser, H. 1934. Rats, lice and history. Boston, MA: Little, Brown and Co. 301 p. 


\section{Appendix A. New Mexico Ectoparasites and Their Mammal Hosts}

\section{Order Siphonaptera (Fleas)}

\section{Family Ceratophyllidae}

Aetheca wagneri Baker, 1904

Bassariscus astutus

Clethrionomys gapperi

Dipodomys merriami

D. ordii

Microtus longicaudus

M. montanus

Mus musculus

Neotoma albigula

$N$. cinerea

N. mexicana

N. micropus

Onychomys leucogaster

Peromyscus boylii

P. leucopus

P. maniculatus

$P$. nasutus

P. truei

Peromyscus sp.

Reithrodontomys megalotis

Sigmodon hispidus

Spermophilus spilosoma

Sylvilagus audubonii

Tamias minimus

T. quadrivitattus

Tamiasciurus hudsonicus

Amaradix bitterootensis Dunn, 1923

Tamiasciurus hudsonicus

Amaradix euphorbae Rothschild, 1905

Clethrionomys gapperi

Peromyscus maniculatus

P. truei

Amphalius necopinus Jordan, 1925

Ochotona princeps

Ceratophyllus vison Baker, 1904

Peromyscus nasutus

Sciurus aberti

Tamias minimus

Tamiasciurus hudsonicus
Eumolpianus eumolpi Rothschild, 1905

Dipodomys merriami

Microtus sp.

Neotoma mexicana

Peromyscus maniculatus

Spermophilus lateralis

Sylvilagus sp.

Tamias minimus

T. quadrivittatus

Tamiasciurus hudsonicus

Thomomys talpoides

Eumolpianus eumolpi americanus Hubbard, 1950

Microtus longicaudus

Sciurus aberti

Spermophilus lateralis

Tamias dorsalis

T. minimus

T. quadrivitattus

Tamiasciurus hudsonicus

Dactylopsylla bluei Fox, 1909

Microtus montanus

Dactylopsylla neomexicana Prince, 1945

Thomomys talpoides

Foxella apachinus C. Fox, 1914

Vulpes macrotis macrotis

Foxella ignotus Baker, 1895

Lynx rufus

Microtus montanus

Mustela frenata

Peromyscus maniculatus

Thomomys bottae

T. talpoides

Urocyon cinereoargenteus

Vulpes macrotis macrotis

Malaraeus sinomus Jordan, 1925

Bassariscus astutus

Dipodomys ordii

Neotoma albigula

$N$. mexicana

Onychomys leucogaster

Peromyscus boylii

P. leucopus

P. maniculatus

$P$. nasutus

Peromyscus sp.

P. truei 
Malaraeus telchinum Rothschild, 1905

Clethrionomys gaperi

Microtus longicaudus

M. montanus

Neotoma cinerea

$N$. mexicana

Neotoma sp.

Onychomys leucogaster

Peromyscus boylii

P. maniculatus

P. truei

Reithrodontomys megalotis

Spermophilus lateralis

Tamiasciurus hudsonicus

Megabothris abantis Rothschild, 1905

Clethrionomys gapperi

Microtus longicaudus

M. montanus

Microtus sp.

Mustela frenata

Neotoma mexicana

Ochotona princeps

Peromyscys maniculatus

P. truei

Spermophilus lateralis

Nosophyllus fasciatus Bosc, 1800

Rattus norvegicus

Opisodasys keeni Baker, 1896

Peromyscus truei

Opisodasys robustus Jordan, 1925

Sciurus aberti

Tamiasciurus hudsonicus

Orchopeas agilis Rothschild, 1905

Neotoma albigula

Onychomys leucogaster

Peromyscus leucopus

Sorex preblei

Vulpes macrotis macrotis

$V$. velox velox

Orchopeas caedens Jordan, 1925

Onychomys arenicola

Peromyscus leucopus

P. truei

Reithrodontomys megalotis

Sciurus aberti

Spermophilus variegatus

Tamiasciurus hudsonicus

Vulpes macrotis

$V$. velox velox

Orchopeas fulleri Traub, 1950

Sciurus arizonensis
Orchopeas leucopus Baker, 1904

Dipodomys merriami

D. ordii

Neotoma albigula

N. micropus

Neotoma sp.

Onychomys lecuogaster

Perognathus flavus

Peromyscus boylii

P. eremicus

P. leucopus

P. maniculatus

P. truei

Reithrodontomys megalotis

Sigmodon hispidus

Spermphilus spilosoma

S. variegatus

Tamias quadrivittatus

Tamiasciurus hudsonicus

Orchopeas sexdentatus Baker, 1904

Dipodomys spectabilis

Neotoma albigula

N. cinerea

N. mexicana

N. micropus

Neotoma sp.

Onychomys leucogaster

Peromyscus maniculatus

P. truei

Sigmodon hispidus

Sylvilagus audubonii

Tamiasciurus hudsonicus

Orchopeas sexdentatus neotomae Augustson, 1943

Bassariscus astutus

Neotoma albigula

$N$. cinerea

N. mexicana

N. micropus

Peromyscus maniculatus

P. truei

Sciurus aberti

Tamiasciurus hudsonicus

Oropsylla hirsutus Baker, 1895

Cynomys gunnisoni

C. ludovicianus

Cynomys sp.

Spermophilus lateralis

S. variegatus

Sylvilagus audubonii

Oropsylla idahoensis Baker, 1904

Cynomys gunnisoni

Microtus longicaudus 
Perognathus flavus

Spermophilus lateralis

S. variegatus

Tamias minimus

T. quadrivattus

Tamiasciurus hudsonicus

Oropsylla labis Jordan and Rothschild, 1922

Cynomys gunnisoni

Oropsylla montanus Baker, 1895

Bassariscus astutus

Cynomys gunnisoni

Dipodomys merriami

Neotoma albigula

Peromyscus truei

Reithrodontomys megalotis

Spermophilus lateralis

S. variegatus

Spilogale gracilis

Sylvilagus sp.

Vulpes macrotis macrotis

Pleochaetis exilis Jordan, 1937

Dipodomys ordii

D. spectabilis

Lepus californicus

Neotoma albigula

N. mexicana

N. micropus

Onychomys leucogaster

Peromyscus leucopus

P. truei

Sigmodon hispidus

Vulpes macrotis macrotis

Spicata bottaceps Hubbard, 1943

Thomomys bottae

Tarsopsyllus coloradensis Baker, 1904

Sciurus aberti

Tamiasciurus hudsonicus

Thrassis aridis Prince, 1944

Bassariscus astutus

Dipodomys ordii

Onychomys leucogaster

Sigmodon hispidus

Thrassis bacchi consimilis Stark, 1957

D. ordii

Neotoma albigula

Onychomys leucogaster

Spermophilus spilosoma

Thrassis campestris Prince, 1944

Dipodomys merriami

D. ordii

D. spectabilis

Neotoma albigula
N. micropus

Neotoma sp.

Onychomys leucogaster

Peromyscus leucopus

$P$. maniculatus

P. truei

Sigmodon hispidus

Spermophilus spilosoma

Sylvilagus audubonii

Thrassis fotus Jordan, 1925

Dipodomys merriami

D. ordii

D. spectabilis

Neotoma albigula

N. micropus

Neotoma sp.

Onychomys leucogaster

Reithrodontomys megalotis

Spermophilus spilosoma

S. variegatus

Sylvilagus audubonii

Thrassis pansus Jordan, 1925

Ammospermophilus harrisii

A. leucurus

Dipodomys ordii

D. spectabilis

Mustela frenata

Neotoma albigula

N. micropus

Onychomys leucopus

Perognathus flavescens

Peromyscus leucopus

P. maniculatus

Reithrodontomys megalotis

Sigmodon hispidus

Spermophilus spilosoma

Thrassis stanfordi Wagner, 1936

Cynomys gunisoni

Marmota flaviventris

Mustela frenata

\section{Family Ctenophthalmidae}

Anomiopsyllus hiemalis Eads and Menzies, 1948

Neotoma albigula

Anomiopsyllus martini Holland, 1965

Sciurus aberti

Anomiopsyllus hiemalis mexicanus Holland, 1965

Dipodomys merriami

Neotoma albigula

N. mexicana

Neotoma sp. 
Peromyscus eremicus

P. maniculatus

P. truei

Spermophilus variegatus

Anomiopsyllusnovomexicanus WilliamsandHoff,1951

Dipodomys merriami

D. ordii

D. spectabilis

Neotoma albigula

N. mexicana

N. micropus

Neotoma sp.

Onychomys leucogaster

Peromyscus boylii

P. leucopus

P. maniculatus

P. truei

Sigmodon hispidus

Sylvilagus audubonii

Sylvilagus sp.

Anomiopsyllus nudata Baker, 1898

Neotoma albigula

N. mexicana

N. micropus

Perognathus flavus

Peromyscus leucopus

P. truei

Sciurus aberti

Spilogale gracilis

Tamias minimus

T. quadrivittatus

Thomomys talpoides

Callistopsyllus terinus Rothschild, 1905

Peromyscus boylii

P. maniculatus

Peromyscus sp.

P. truei

Catallagia charlottensis Baker, 1898

Peromyscus boylii

Catallagia decipiens Rothschild, 1915

Clethrionomys gapperi

Microtus lonigcaudus

M. montanus

Microtus sp.

Neotoma cinerea

N. mexicana

Peromyscus maniculatus

P. truei

Spermophilus lateralis

Tamias quadrivitattus

Tamiasciurus hudsonicus
Catallagia neweyi Holland and Loshbaugh, 1958

Spermophilus tridecemlineatus

Corrodopsylla curvata Rothschild, 1915

Microtus montanus

Sorex cinereus

Delotelis telegoni Jordan, 1937

Microtus longicaudus

Epitedia stanfordi Traub, 1944

Bassariscus astutus

Neotoma albigula

N. micropus

Onychomys leucogaster

Perognathus flavus

Peromyscus leucopus

P. maniculatus

P. nasutus

P. truei

Reithrodontomys megalotis

Tamias quadrivittatus

Epitedia wenmanni Rothschild, 1904

Peromyscus sp.

P. truei

Sigmodon hispidus

Megarthroglossus bisetis Jordan and Rothschild, 1915

Bassariscus astutus

Dipodomys spectabilis

Microtus longicaudus

Neotoma albigula

$N$. cinerea

N. mexicana

N. micropus

Neotoma sp.

Ochotona princeps

Onychomys leucogaster

Peromyscus boylii

P. leucopus

P. maniculatus

$P$. nasutus

Peromyscus sp.

P. truei

Reithrodontomys egalotis

Sigmodon hispidus

Sylvilagus audubonii

S. nuttalli

Sylvilagus sp.

Tamiasciurus hudsonicus

MegarthroglossuscavernicolusMendezandHaas,1972 Neotoma cinerea

Megarthroglossus divisus Baker, 1898

Neotoma albigula

$N$. cinerea

N. micropus 
Ochotona princeps

Onychomys leucogaster

Peromyscus boylii

P. leucopus

P. maniculatus

Peromyscus sp.

P. truei

Tamiasciurus hudsonicus

Megarthroglossu procus Jordan and Rothschild, 1915

Neotoma albigula

Megarthroglossus wilsoni Mendez and Haas, 1973

Tamias minimus

Meringis altipecten Traub and Hoff, 1951

Dipodomys merriami

D. ordii

D. spectabilis

Neotoma albigula

Onychomys arenicola

O. leucogaster

Peromyscus boylii

P. maniculatus

Meringis arachis Jordan, 1929

Bassariscus astutus

Dipodomys merriami

D. ordii

D. spectabilis

Neotoma albigula

Onychomys leucogaster

Perognathus flavescens

P. flavus

Peromyscus maniculatus

Meringis bilsingi Eads and Menzies, 1949

Dipodomys merriami

D. ordii

D. spectabilis

Neotoma micropus

Neotoma sp.

Onychomys leucogaster

Peromyscus leucopus

$P$. maniculatus

Reithrodontomys megalotis

Sigmodon hispidus

Sylvilagus audubonii

Meringis dipodomys Kohls, 1938

Dipodomys merriami

D. ordii

D. spectabilis

Neotoma micropus

Neotoma sp.

Onychomys leucogaster

Perognathus flavus

Sylvilagus audubonii
Sylvilagus sp.

Meringis disparilis Eads, 1979

Dipodomys merriami

D. ordii

Onychomys leucogaster

Meringis facilis Eads, 1979

Neotoma micropus

Onychomys leucogaster

Perognathus flavus

Meringis jamesoni Hubbard, 1943

Dipodomys spectabilis

Neotoma albigula

Onychomys leucogaster

Perognathus flavus

Spermophilus spilosoma

Meringis nidi Williams and Hoff, 1951

Dipodomys merriami

D. ordii

D. spectabilis

Neotoma albigula

N. micropus

Neotoma sp.

Onychomys leucogaster

Perognathus flavus

Peromyscus leucopus

P. maniculatus

P. truei

Reithrodontomys megalotis

Sigmodon hispidus

Spermophilus spilosoma

Sylvilagus audubonii

Sylvilagus sp.

Meringis parkeri Jordan, 1937

Cynomys sp.

Dipodomys merriami

D. ordii

D. spectabilis

Microtus sp.

Neotoma albigula

N. micropus

Onychomys leucogaster

Perognathus flavus

Peromyscus maniculatus

Spermophilus spilosoma

Meringis rectus Morlan, 1953

Cynomys gunnisoni

Dipodomys merriami

D. ordii

D. spectabilis

Neotoma albigula

N. micropus

Neotoma sp. 
Onychomys leucogaster

Perognathus flavus

Peromyscus leucopus

P. maniculatus

P. truei

Reithrodontomys megalotis

Sigmodon hispidus

Spermophilus spilosoma

S. variegatus

Sylvilagus audubonii

Sylvilagus sp.

Phalacropsylla allos Wagner, 1936

Neotoma cinerea

N. mexicana

Peromyscus maniculatus

P. truei

Phalacropsylla hamata Tipton and Mendez, 1968

Neotoma albigula

Peromyscus leucopus

Phalacropsylla morlani Eads and Campos, 1982

Ochotona princeps

Rhadinopsylla fraterna Baker, 1895

Clethrionomys gapperi

Dipodomys merriami

D. spectabilis

Neotoma cinerea

Neotoma sp.

Peromyscus leucopus

Sigmodon hispidus

Sylvilagus audubonii

Sylvilagus sp.

Rhadinopsylla goodi Hubbard, 1941

Bassariscus astutus

Rhadinopsylla multidenticulatus Morlan and

Prince, 1954

Dipodomys merriami

D. ordii

D. spectabilis

Neotoma albigula

N. micropus

Onychomys leucogaster

Peromyscus leucopus

Sigmodon hispidus

Sylvilagus audubonii

Rhadinopsylla sectilis Jordan and Rothschild, 1923

Neotoma albigula

$N$. cinerea

Peromyscus boylii

P. leucopus

P. maniculatus

P. nasutus

P. truei
Reithrodontomys megalotis

Stenistomera alpina Baker, 1895

Bassariscus astutus

Lynx rufus

Mustela frenata

Neotoma albigula

$N$. cinerea

N. mexicana

Ochotona princeps

Peromyscus truei

Sylvilagus nuttallii

Tamias quadrivittatus

Vulpes macrotis macrotis

Stenistomera macrodactyla Good, 1942

Neotoma albigula

Peromyscus truei

Stenoponia americana Baker, 1899

Peromyscus maniculatus

P. nasutus

Stenoponia ponera Traub and Johnson, 1952

Peromyscus boylii

\section{Family Hystrichopsyllidae}

Atyphloceras echis Jordan and Rothschild, 1915

Bassariscus astutus

Neotoma albigula

N. micropus

Neotoma sp.

Peromyscus boylii

P. maniculatus

P. nasutus

Peromyscus sp.

P. truei

Atyphloceras multidentatus C. Fox, 1909

Peromyscus boylii

Hystrichopsylla dippiei Rothschild, 1902

Microtus longicaudus

M. montanus

Neotoma cinerea

N. mexicana

Peromyscus maniculatus

P. nasutus

P. truei

Spermophilus lateralis

Tamias minimus

T. quadrivittatus

Tamiasciurus hudsonicus

\section{Family Ischnopsyllidae}

Myodopsylla gentilis Jordan and Rothschild, 1921

Myotis yumanensis 
Myodopsylla nordina Traub and Hoff, 1951

Myotis thysanodes

Sternopsylla texanus Fox, 1914

Tadarida brasiliensis

\section{Family Leptopsyllidae}

Ctenophyllus armatus Wagner, 1901

Ochotona princeps

Thomomys talpoides

Odontopsyllus dentatus Baker, 1904

Sylvilagus audubonii

S. nuttalli

Peromyscopsylla adelpha Rothschild, 1915

Dipodomys ordii

Microtus longicaudus

Onychomys leucogaster

Peromyscus boylii

P. leucopus

P. maniculatus

P. nasutus

P. truei

Reithrodontomys megalotis

Tamias quadrivittatus

Peromyscopsylla draco Hopkins, 1951

Neotoma micropus

Onychomys leucogaster

Peromyscus leucopus

P. maniculatus

Reithrodontomys megalotis

Sigmodon hispidus

Peromyscopsylla hamifer vigens Jordan, 1937

Microtus longicaudus

M. montanus

M. pennsylvanicus

Peromyscus maniculatus

Peromyscopsylla hesperomys Baker, 1904

Neotoma albuigula

Onychomys leucogaster

Peromyscus leucopus

$P$. maniculatus

P. nasutus

P. truei

Peromyscopsylla selenis Rothschild, 1906

Microtus longicaudus

M. montanus

M. pennsylvanicus

Peromyscus maniculatus

\section{Family Pulicidae}

\section{Ctenocephalides felis Bouche, 1835}

Canis familiaris
Odocoileus hemionus

Echidnophaga gallinaceus Westwood, 1875

Bassariscus astutus

Cynomys gunnisoni

Dipodomys merriami

D. ordii

D. spectabilis

Felis catus

Lepus californicus

Mephitis sp.

Neotoma albigula

N. micropus

Neotoma sp.

Onychomys leucogaster

Peromyscus leucopus

P. truei

Rattus norvegicus

Reithrodontomys megalotis

Spermophilus spilosoma

$S$. variegatus

Sylvilagus audubonii

Sylvilagus sp.

Urocyon cinereoargenteus

Vulpes velox velox

Euhoplopsyllus affinis Baker, 1904

Dipodomys merriami

D. spectabilis

Lepus californicus

Neotoma sp.

Perognathus flavus

Peromyscus truei

Sigmodon hispidus

Spermophilus spilosoma

Sylvilagus audubonii

S. floridanus

S. nuttalli

Sylvilagus sp.

Urocyon cinereoargenteus

Vulpes macrotis macrotis

$V$. velox velox

$V$. vulpes

Euhoplopsyllus glacialis Taschenberg, 1880

Canis familiaris

C. latrans

Felis catus

Lepus californicus

Lynx rufus

Sigmodon hispidus

Sylvilagus audubonii

Hoplopsyllus anomalus Baker, 1904

Cynomys gunnisoni

Lepus californicus 
Neotoma albigula

Peromyscus boylii

Spermophilus spilosoma

S. variegatus

Spilogale gracilis

Sylvilagus sp.

Pulex irritans Linnaeus, 1758

Canis familiaris

Dipodomys spectabilis

Felis catus

Homo sapiens

Sylvilagus audubonii

Sylvilagus sp.

Urocyon cinereoargenteus

Vulpes macrotis

V. velox

Pulex simulans Baker, 1895

Bassariscus astutus

Cynomys ludovicianus

Dipodomys spectabilis

Sylvilagus audubonii

Urocyon cinereoargenteus

Vulpes macrotis macrotis

$V$. velox velox

V. vulpes

Spilopsyllus inaequalis Baker, 1895

Canis familiaris

C. latrans

Felis catus

Lepus californicus

Lynx rufus

Ochotona princeps

Sylvilagus audubonii

S. floridanus

Urocyon cineroaregenteus

Vulpes macrotis macrotis

V. vulpes

Xenopsylla cheopis Rothschild, 1903

Rattus norvegicus

R. rattus

\section{Family Rhopalopsyllidae}

Polygenis gwyni C. Fox, 1914

Dipodomys spectabilis

Neotoma micropus

Neotoma sp.

Sigmodon hispidus

Sylvilagus audubonii

Sylvilagus sp.

Polygenis martinezbaezi Vargas, 1951

Sigmodon ochrognathus

\section{Order Anoplura (Sucking Lice)}

Enderleinellus suturalis Osborn, 1891

Ammospermophilus harrisii

Cynomys gunnisoni

Spermophilus spilosoma

Fahrenholzia pinnata Kellogg and Ferris, 1915

Chaetodipus intermedius

Dipodomys merriami

D. ordii

D. spectabilis

Onychomys leucogaster

Perognathus flavus

Fahrenholzia zacatecae Ferris, 1922

Chaetodipus hispidus

Haemodipsus setoni Ewing, 1924

Lepus californicus

Sylvilagus audubonii

Haematopinus asini Linnaeus, 1758

Equus asinus

Equus caballus

Dipodomys merriami

Hoplopleura acanthopus Burmeisterm 1839

Dipodomys spectabilis

Microtus longicaudus

Hoplopleura arboricola Kellogg and Ferris, 1915

Dipodomys ordii

D. spectabilis

Tamias quadrivittatus

Hoplopleura arizonensis Stojanovich and Pratt, 1961 Sigmodon hispidus

Hoplopleura ferrisi Cook and Beer, 1959

Dipodomys ordii

Peromyscus eremicus

Hoplopleura hesperomydis Osborn, 1891

Dipodomys ordii

Microtus longicaudus

Onychomys leucogaster

Peromyscus boylii

P. leucopus

P. maniculatus

$P$. nasutus

P. truei

Hoplopleura hirsuta Ferris, 1916

Dipodomys ordii

Onychomys leucogaster

Sigmodon hispidus

Sylvilagus audubonii

Hoplopleura onychomydis Cook and Beer, 1959

Onychomys leucogaster

Hoplopleura reithrodontomydis Ferris, 1951

Reithrodontomys megalotis 
Linognathus africanus Kellogg and Paine, 1911

Capra hircus

Ovis aries

Linognathus setosus von Olfers, 1816

Canis familiaris

Canis latrans

Linognathoides citellinus Ferris, 1942

Cynomys gunnisoni

Linognathoides laeviusculus Grube, 1851

Spermophilus variegatus

Linognathoides marmotae Ferris, 1923

Marmota flaviventris

Linognathoides neotomae Ferris, 1942

Neotoma sp.

Linognathoides spilosomae Stojanovich and Pratt, 1961

Spermophilus spilosoma

Neohaematopinus citellinus Ferris, 1942

Cynomys gunnisoni

Spermophilus spilosoma

S. variegatus

Neohaematopinus neotomae Ferris, 1941

Dipodomys ordii

Neotoma albigula

N. mexicana

N. micropus
Neohaematopinus pacificus Kellogg and Ferris, 1915 Tamias quadrivittatus

Neohaematopinus spilosomae Stojanovich and Pratt, 1961

Spermophilus spilsoma

Pediculus humanus Linnaeus, 1758

Homo sapiens

Phthirus pubis Linnaeus, 1758

Homo sapiens

Polyplax auricularis Kellogg and Ferris, 1915

Onychomys leucogaster

Peromyscus maniculatus

P. nasutus

P. truei

Order Mallophaga (Chewing Lice)

Goniodes squamatus Emerson, 1950

Dipodomys ordii

Werneckiella (Bovicola) equi Denny, 1842

Equus asinus

E. caballus 


\section{Appendix B.}

\section{New Mexico County Records of Fleas (Siphonaptera)}

\section{Bernalillo}

Aetheca wagneri

Anomiopsyllus novomexicanensis

A. hiemalis mexicanus

Atyphloceras echis

Catallagia decipiens

Ctenocephalides felis

Echidnophaga gallinaceus

Epitedia stanfordi

Euhoplopsyllus affinis

Eumolpianus eumolpi

Hoplopsyllus anomalus

Hystrichopsylla dippiei

Foxella ignotus

Malaraeus sinomus

Megarthroglossis bisetis

M. divisus

M. arachis

M. bilsingi

M. facilis

M. nidi

M. parkeri

$M$. rectus

Myodopsylla nordina

Nosopsyllus fasciatus

Orchopeas sexdentatus

O. sexdentatus neotomae

Oropsylla hirsutus

O. montanus

Peromyscopsylla hesperomys

Phalacropsylla allos

P. hamata

Pulex irritans

Pulex simulans

Rhadinopsylla goodi

$R$. sectilis

Spilopsyllus inaequalis

Stenistomera alpina

Thrassis aridis

T campestris

T. pansus

Thrassis stanfordi

Xenopsyllus cheopis

\section{Catron}

Anomiopsyllus hiemalis mexicanus
A. martini
Ceratophyllus vison
Meringis parkeri
Opisodasys robustus
Orchopeas caedens
O. fulleri
Oropsylla hirsutus
O. idahoensis

\section{Chaves}

Aetheca wagneri

Anomiopsyllus hiemalis mexicanus

A. novomexicanensis

Atyphloceras echis

Echidnophaga gallinaceus

Epitedia wenmanni

Euhoplopsyllus affinis

E. glacialis

Malaraeus telchinum

Megarthroglossus bisetis

M. bilsingi

M. dipodomys

M. facilis

M. nidi

M. rectus

Orchopeas agilis

O. caedens

O. leucopus

O. sexdentatus

O. sexdentatus neotomae

Oropsylla hirsutus

Peromyscopsylla draco

Polygenis gwyni

Pulex irritans

P. simulans

Rhadinopsylla fraterna

$R$. multidenticulatus

Spilopsylla inaequalis

Thrassis aridis

T. bacchi consimilis

T. campestris

T. fotus

T. pansus

\section{Cibola}

None recorded. 


\section{Colfax}

Anomiopsyllus hiemalis mexicanus

Catallagia neweyi

Malaraeus telchinum

Megabothris megacolpus

Megarthroglossus bisetis

M. divisus

M. wilsoni

Oropsylla tuberculatus cynomuris

O. idahoensis

O. labis

Pleochaetis exilis

\section{Curry}

Echidnophaga gallinaceus

Euhoplopsyllus affinis

E. glacialis

\section{DeBaca}

Pleochaetis exilis

Pulex irritans

\section{Dona Ana}

Anomiopsyllus novomexicanensis

Euhoplopsyllus affinis

Meringis altipectin

M. arachis

M. bilsingi

M. disparilis

$M$. nidi

M. rectus

Orchopeas agilis

\section{Eddy}

Anomiopsyllus hiemalis

Echidnophaga gallinaceus

Meringis altipectin

M. disparilis

M. nidi

Orchopeas caedens

Sternopsylla texanus

Thrassis fotus

\section{Grant}

Atyphloceras echis

Echidnophaga gallinaceus

Euhoplopsyllus affinis

Meringis arachis

M. dipodomys

Spilopsyllus inaequalis

Stenoponia ponera

\section{Guadalupe}

Megarthroglossus bisetis

\section{Harding}

None recorded.

\section{Hidalgo}

Anomiopsyllus novomexicanensis

Atyphloceras echis

Echidnophaga gallinaceus

Meringis altipectin

M. arachis

M. nidi

Polygenis martinezbaezi

Thrassis aridis

Thrassis pansus

Xenopsylla cheopis

\section{Lea}

Anomiopsyllus hiemalis

Echidnophaga gallinaceus

Euhoplopsyllus affinis

E. glacialis

Meringis bilsingi

M. dipodomys

M. nidi

M. parkeri

Orchopeas agilis

O. caedens

O. sexdentatus

Pulex irritans

P. simulans

Thrassis fotus

\section{Lincoln}

Anomiopsyllus novomexicanensis

A. hiemalis mexicanus

Megarthroglossus divisus

Meringis rectus

Peromyscopsylla adelpha

\section{Los Alamos}

Aetheca wagneri

Catallagia decipiens

Ceratophyllus vison

Malaraeus telchinum

Megarthroglossus bisetis

Peromyscopsylla adelpha

Pulex irritans

\section{Luna}

Anomiopsyllus novomexicanensis

Echidnophaga gallinaceus

Meringis altipectin

M. arachis

M. bilsingi

M. disparilis

Pulex irritans

\section{McKinley}

Eumolpianus eumolpi americanus

Foxella apachinus

Megarthroglossus bisetis 
M. divisus

Orchopeas agilis

O. caedens

Oropsylla montanus

Phalacropsylla hamata

Pleochaetis exilis

Pulex irritans

Spilopsyllus inaequalis

Stenistomera alpina

\section{Mora}

None recorded.

\section{Otero}

Anomiopsyllus novomexicanensis

Eumolpianus eumolpi

E. eumolpi americanus

Euhoplopsyllus affinis

Megarthroglossus divisus

Meringis bilsingi

M. nidi

$M$. rectus

Orchopeas caedens

O. leucopus

Oropsylla montanus

\section{Quay}

None recorded.

\section{Rio Arriba}

Aetheca wagneri

Anomiopsyllus heimalis mexicanus

A. novomexicanensis

Callistopsyllus terinus

Catallagia decipiens

Echidnophaga gallinaceus

Epitedia wenmanni

Euhoplopsyllus affinis

Eumolpianus eumolpi

E. eumolpi americanus

Foxella ignotus

Malaraeus telchinum

Megabothris abantis

Megarthroglossus bisetis

M. divisus

Meringis parkeri

Opisodasys keeni

Orchopeas leucopus

O. sexdentatus

O. sexdentatus neotomae

Oropsylla hirsutus

O. idahoensis

O. tuberculatus cynomuris

Peromyscopsulla adelpha

Pulex irritans

Spilopsyllus inaequalis
Thrassis pansus

T. stanfordi

\section{Roosevelt}

Euhoplopsyllus affinis

E. glacialis

Meringis bilsingi

Orchopeas leucopus

Oropsylla hirsutus

Peromyscopsylla hesperomys

Pleochaetis exilis

Pulex irritans

$P$. simulans

Thrassis aridis

T. fotus

\section{San Juan}

Amaradix euphorbae

Anomiopsyllus novomexicanensis

Meringis parkeri

$M$. rectus

Pleochaetis exilis

Pulex simulans

Spilopsylla inaequalis

\section{San Miguel}

Aetheca wagneri

Atyphloceras echis

Callistopsyllus teinus

Dactylopsylla bluei

D. neomexicana

Megarthroglossus bisetis

Peromyscopsylla hamifer vigens

P. selenis

\section{Sandoval}

Aetheca wagneri

Amaradix bitterootensis

A. vonfintelis

Anomiopsyllus nudata

Atyphloceras echis

Callistopsylla terinus

Catallagia decipiens

Ceratophyllus vison

Corrodopsylla curvata

Echidnophaga gallinaceus

Epitedia stanfordi

Euhoplopsyllus affinis

E. glacialis

Eumolpianus eumolpi americanus

Foxella ignotus

Hoplopsyllus anomalus

Hystrichopsylla dippiei

Malaraeus sinomus

M. telchinum

Megabothris abantis 
Megarthroglssus bisetis

M. cavernicolus

M. procus

Meringis bilsingi

M. dipodomys

M. jamesoni

M. parkeri

M. rectus

Odontopsyllus dentatus

Opisodasys robustus

Orchopeas agilis

O. caedens

O. leucopus

O. sexdentatus

O. sexdentatus neotomae

Oropsylla hirsutus

O. idahoensis

O. montanus

O. tuberculatus cynomuris

Peromyscopsylla adelpha

P. draco

P. hamifer vigens

$P$. hesperomys

P. selenis

Phalacropsylla allos

Pulex irritans

Rhadinopsylla fraterna

$R$. sectilis

Spicata rara

Spilopsyllus inaequalis

Stenistomera alpina

S. macrodactyla

Stenoponia Americana

Tarsopsylla coloradensis

Thrassis campestris

T. pansus

\section{Santa Fe}

Aetheca wagneri

Amaradix euphorbae

Amphalius necopinus

Anomiopsyllus hiemalis mexicanus

A. nudata

Atyphloceras echis

A. multidentatus multidentatus

Callistopsylla terinus

Catallagia decipiens

C. charlottensis

Ceratophyllus vison

Ctenocephalides felis

Ctenophyllus armatus

Delotelis telegoni

Echidnophaga gallinaceus
Epitedia stanfordi

Euhoplopsyllus affinis

Eumolpianus eumolpi americanus

Foxella ignotus

Hoplopsyllus anomalus

Hystrichopsylla dippiei

Malaraeus sinomus

M. telchinum

Megabothris abantis

Megarthroglssus bisetis

M. divisus

Meringis arachis

M. jamesoni

M. nidi

M. parkeri

$M$. rectus

Myodopsylla gentilis

Odontopsyllus dentatus

Orchopeas agilis

O. caedens

O. leucopus

O. sexdentatus

O. sexdentatus neotomae

Oropsylla hirsutus

O. idahoensis

O. montanus

O. tuberculatus cynomuris

Peromyscopsylla adelpha

P. draco

P. hamifer vigens

P. selenis

Phalacropsylla morlani

Pleochaetis exilis

Pulex irritans

Rhadinopsylla fraterna

$R$. multidenticulatus

$R$. sectilis

Spicata bottaceps

Spilopsyllus inaequalis

Stenistomera alpina

S. macrodactyla

Tarsopsylla coloradensis

Thrassis aridis

T. bacchi consimilis

T. campestris

T. fotus

T. pansus

T. stanfordi

Sierra

Anomiopsyllus novomexicanensis

Meringis altipectin

M. arachis 
M. bilsingi

M. parkeri

\section{Socorro}

Anomiopsyllus hiemalis mexicanus

A. novomexicanensis

Ctenophthalmus pseudagyrtes

Echidnophaga gallinaceus

Euhoplopsyllus affinis

Eumolpianus eumolpi

Foxella ignotus

Hystrichopsylla dippei

Malaraeus sinomus

Megabothris abantis

Meringis altipectin

M. arachis

M. parkeri

Opisodasys robustus

Orchopeas agilis

O. caedens

O. leucopus

O. sexdentatus neotomae

O. montanus

Pleochaetis exilis

Plusaetis equatorius asetus

Pulex irritans

P. simulans

Spilopsyllus inaequalis

Thrassis bacchi consimilis

T. pansus

\section{Taos}

Anomiopsylla hiemalis mexicanus

Ceratophyllus vison

Opisodasy robustus

Oropsylla hirsutus

\section{Torrance}

Epitedia wenmanni

Euhoplopsyllus affinis

Meringis rectus

Oropsylla hirsutus

Pulex irritans

Thrassi pansus

\section{Union}

Aetheca wagneri

Malaraerus sinomus

Peromyscopsylla adelpha

P. draco

Pleochaetis exilis

Pulex irritans

\section{Valencia}

Anomiopsyllus hiemalis mexicanus A. novomexicanensis

Megarthroglossus bisetis
Meringis facilis

M. rectus

Nosopsyllus fasciatus

Peromyscopsylla adelpha 


\section{New Mexico County Records of Lice Order Anoplura and Order Mallophaga (as noted)}

\section{Bernalillo}

Enderleinellus suturalis

Fahrenholzia pinnata

F. zacatecae

Goniodes squamatus (O. Mallophaga)

Hoplopleura arboricola

H. onychomydis

H. hesperomydis

Linognathus africanus

L. setosus

Linognathoides laeviusculus

L. neotomae

L. spilosomae

Neohaematopinus citellinus

N. neotomae

N. spilosomae

Pediculus humanus

Phthirus pubis

Polyplax auricularis

\section{Catron}

Fahrenholzia zacatecae

\section{Chaves}

Fahrenholzia zacatecae

\section{Cibola}

Fahrenholzia zacatecae

\section{Colfax}

Fahrenholzia zacatecae

\section{Curry}

Fahrenholzia zacatecae

Haemodipsus setoni

\section{DeBaca}

Fahrenholzia zacatecae

\section{Dona Ana}

Fahrenholzia zacatecae

\section{Eddy}

Fahrenholzia zacatecae

\section{Grant}

Fahrenholzia zacatecae

Hoplopleura ferrisi

\section{Guadalupe}

Fahrenholzia zacatecae

\section{Harding}

Fahrenholzia zacatecae

\section{Hidalgo}

Fahrenholzia zacatecae

\section{Lea}

Fahrenholzia zacatecae

\section{Lincoln}

Fahrenholzia zacatecae

\section{Los Alamos}

Fahrenholzia zacatecae

\section{Luna}

Fahrenholzia zacatecae

McKinley

Fahrenholzia zacatecae

Mora

Fahrenholzia zacatecae

\section{Otero}

Fahrenholzia pinnata

Haematopinus asini

Werneckiella (Bovicola) equi (O. Mallophaga)

Quay

Fahrenholzia zacatecae

\section{Rio Arriba}

Fahrenholzia zacatecae

\section{Roosevelt}

Fahrenholzia pinnata

Fahrenholzia zacatecae

Hoplopleura hesperomydis

H. hirsuta

Polyplax auricularis

\section{San Juan}

Fahrenholzia zacatecae

\section{San Miguel}

Fahrenholzia zacatecae

\section{Sandoval}

Fahrenholzia zacatecae

Hoplopleura hesperomydis

\section{Santa Fe}

Enderleinellus suturalis

Fahrenholzia zacatecae

Hoplopleura acanthopus

H. arboricola

H. hesperomydis

Linognathoides citellinus

L. laeviusculus

L. marmotae

L. neotomae

L. pacificus

Neohaematopinus citellinus

N. neotomae 
N. pacificus

Polyplax auricularis

\section{Sierra}

Fahrenholzia zacatecae

\section{Socorro}

Fahrenholzia zacatecae

Hoplopleura arboricola

$H$. arizonensis

H. reithrodontomydis

\section{Taos}

Fahrenholzia zacatecae Hoplopleura reithrodontomydis

\section{Torrance}

Fahrenholzia zacatecae

\section{Union}

Fahrenholzia zacatecae

\section{Valencia}

Fahrenholzia zacatecae 


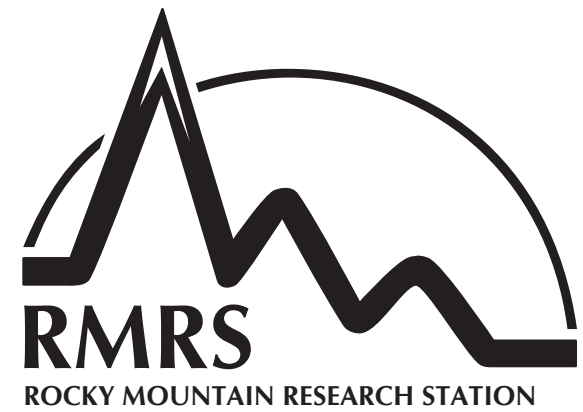

The Rocky Mountain Research Station develops scientific information and technology to improve management, protection, and use of the forests and rangelands. Research is designed to meet the needs of the National Forest managers, Federal and State agencies, public and private organizations, academic institutions, industry, and individuals.

Studies accelerate solutions to problems involving ecosystems, range, forests, water, recreation, fire, resource inventory, land reclamation, community sustainability, forest engineering technology, multiple use economics, wildlife and fish habitat, and forest insects and diseases. Studies are conducted cooperatively, and applications may be found worldwide.

\section{Research Locations}

Flagstaff, Arizona

Fort Collins, Colorado*

Boise, Idaho

Moscow, Idaho

Bozeman, Montana

Missoula, Montana

Lincoln, Nebraska
Reno, Nevada

Albuquerque, New Mexico

Rapid City, South Dakota

Logan, Utah

Ogden, Utah

Provo, Utah

Laramie, Wyoming

*Station Headquarters, Natural Resources Research Center, 2150 Centre Avenue, Building A, Fort Collins, CO 80526.

The U.S. Department of Agriculture (USDA) prohibits discrimination in all its programs and activities on the basis of race, color, national origin, sex, religion, age, disability, political beliefs, sexual orientation, or marital or family status. (Not all prohibited bases apply to all programs.) Persons with disabilities who require alternative means for communication of program information (Braille, large print, audiotape, etc.) should contact USDA's TARGET Center at (202) 720-2600 (voice and TDD).

To file a complaint of discrimination, write USDA, Director, Office of Civil Rights, Room 326 W, Whitten Building, 1400 Independence Avenue, SW, Washington, D.C. $20250-9410$ or call (202) 720-5964 (voice and TDD). USDA is an equal opportunity provider and employer. 